\title{
GLOBAL EXISTENCE OF A WEAK SOLUTION OF THE INCOMPRESSIBLE EULER EQUATIONS WITH HELICAL SYMMETRY AND $L^{p}$ VORTICITY
}

\author{
A. C. BRONZI, M. C. LOPES FILHO, AND H. J. NUSSENZVEIG LOPES
}

MSC Subject Classifications: 35Q31, 76B47.

Keywords: Helical symmetry, Euler equations, vorticity.

\begin{abstract}
We prove the global existence of a helical weak solution of the 3D Euler equations, in full space, for an initial velocity with helical symmetry, without swirl and whose initial vorticity is compactly supported in the axial plane and belongs to $L^{p}$, for some $p>\frac{4}{3}$. This result is an extension of the existence part of the work of B. Ettinger and E. Titi, [9], who studied well-posedness of the Euler equations with helical symmetry without swirl, with bounded initial vorticity, in a helical pipe.
\end{abstract}

\section{INTRODUCTION}

Consider the 3D incompressible Euler equations, describing the motion of an ideal incompressible fluid in $\mathbb{R}^{3}$ with initial velocity $u^{0}=u^{0}(x)$,

$$
\left\{\begin{array}{l}
\partial_{t} u+(u \cdot \nabla) u+\nabla p=0 \\
\operatorname{div} u=0 \\
u(x, 0)=u^{0}(x) .
\end{array}\right.
$$

Above, $u=u(t, x) \in \mathbb{R}^{3}$ is the velocity field, $p=p(t, x) \in \mathbb{R}$ is the scalar pressure and $(t, x) \in$ $\mathbb{R} \times \mathbb{R}^{3}$.

Another physically relevant dynamic variable for incompressible flows is $\omega=\omega(t, x)=\operatorname{curl} u(t, x)$, the vorticity, which satisfies the evolution equation:

$$
\partial_{t} \omega+(u \cdot \nabla) \omega=(\omega \cdot \nabla) u .
$$

Existence of global smooth solutions for the incompressible 3D Euler equations with smooth initial data is an important open problem, with a large associated literature. Local (in time) existence with smooth initial data is known, see [14. Global existence of a weak solution has only recently been established by E. Wiedemann in [22] in the context of wild solutions, see also [5], for arbitrary initial divergence-free velocity in $L^{2}$. The vorticity associated with wild solutions does not, in principle, satisfy equation (1.2), so that another important open problem is the global existence of a weak solution of equation (1.1) whose vorticity is also a weak solution of equation (1.2); we will refer to such solutions as tame weak solutions.

In contrast, in dimension two, the Euler equations are globally well-posed for smooth initial data, see [18. This distinction is usually attributed to the term $(\omega \cdot \nabla) u$, in equation (1.2), responsible for vortex stretching; this term vanishes identically for $2 \mathrm{D}$ flows. One way to explore the gap between $2 \mathrm{D}$ and $3 \mathrm{D}$ flows is to consider flows with more complicated symmetries, such as axial symmetry and also helical symmetry. There is a large literature devoted to axisymmetric Euler flows, which naturally breaks down into two cases: axisymmetric flows without swirl, and the general case, with swirl. For axisymmetric flows without swirl, global well-posedness of smooth solutions is due to A. Majda and to X. St. Raymond, see [17, 19, 12]. Global existence of tame weak solutions was first proved by D. Chae and N. Kim for initial vorticity in $L^{p}, p>6 / 5$ in [4]. This result was later improved to "near-vortex-sheet" data in [3]. For vortex sheet initial data the problem of existence remains open, but in [7, J.-M. Delort showed that the argument used to obtain existence for vortex sheet initial data in 2D could not be extended to axisymmetric flows without swirl, and further analysis of concentrations was performed in [13. For axisymmetric flows with swirl, and 
for general flows without any particular symmetry, the focus has been on criteria and scenarios for blow-up, see [1, 2] and references therein.

Helical flows are flows invariant under a one-dimensional group of rigid motions of Euclidean space generated by a simultaneous rotation around an axis and a translation along the same axis. Although as common in practice as axisymmetric flows, helical flows have received a great deal less mathematical attention. The literature specific to inviscid flows with helical symmetry reduces to two articles, namely 8, where A. Dutrifoy proved global well-posedness for smooth initial data and 9, where B. Ettinger and E. Titi established global existence and uniqueness of weak solutions with bounded initial vorticity; in both papers a geometric condition, analogous to the no swirl hypothesis for axisymmetric flows, is assumed. In general, helical flows have a more decided 2D nature than axisymmetric flows; for example, global existence for helical NavierStokes is known, see [16], whereas this same problem is open for axisymmetric Navier-Stokes. One reason that helical flows have received less attention might be that the helical symmetry reduction is algebraically more complicated. The present article is part of a research programme aimed at investigating incompressible helical flows with a view towards both the inviscid singularity problem, and the mathematical treatment of weak solutions. Specifically, our main result in this article is an analogue of the $L^{p}$ axisymmetric existence result of Chae and Kim; we obtain the critical exponent $4 / 3$ for helical flows in the full space. Previous work on helical flow has focused on bounded helical domains; we choose to carry out our analysis in the full space. Although this choice creates technical complications which we are forced to address here, the full space case is conceptually simpler, and can be connected in a more physically natural way with small viscosity flows.

This article is organized as follows. In Section 2 we fix notation, introduce basic definitions and we state some well-known results. In Section 3 we develop basic tools to treat full space helical flows. More precisely, in Section 3.1 we derive a formula for the Green's function for the Laplacian in $\mathbb{R}^{3}$, periodic in the $x_{3}$-direction, which is used to write explicitly the relevant Biot-Savart law. In Section 3.2 we introduce the definition of weak solution and in Section 3.3 we state and prove a local well-posedness result for smooth solutions to problem (1.1). Finally, in sections 4 and 5 we prove two versions of the main theorem, first assuming the integral of the third component of vorticity vanishes, which implies velocity vanishes at infinity and greatly simplifies the analysis, and then, second, without this hypothesis.

\section{Preliminaries}

Our purpose in this section is to fix notation, introduce the basic definitions and to recall some known results, taken mainly from [9], for convenience of the reader.

Let $\kappa$ be a positive constant and define $\Omega:=\mathbb{R}^{2} \times(-\pi \kappa, \pi \kappa)$. We denote by $\tilde{x}$ the first two components of $x \in \mathbb{R}^{3}$, i.e., if $x=\left(x_{1}, x_{2}, x_{3}\right)$ then $\tilde{x}=\left(x_{1}, x_{2}\right)$. Let $L_{p e r}^{p}(\Omega)$ and $H_{p e r}^{n}(\Omega)$ denote the spaces of functions $f=f(x), x \in \mathbb{R}^{3}$, which are periodic in the $x_{3}$-direction with period $2 \kappa \pi$ and which belong, respectively, to $L^{p}(\mathcal{O})$ and $H^{n}(\mathcal{O})$, for all open sets of the form $\mathcal{O}=\mathbb{R}^{2} \times I$, with $I \subset \mathbb{R}$ a bounded open interval. Let $V_{p e r}^{n}(\Omega)$ be the space of all vector-valued functions in $H_{p e r}^{n}(\Omega)$ which are divergence free and let $L_{c, p e r}^{p}(\Omega)$ denote the space of functions in $L_{\text {per }}^{p}(\Omega)$ which are compactly supported in $\tilde{x}$, i.e. such that, for almost all $x_{3} \in \mathbb{R}$, the support of $f\left(\cdot, x_{3}\right)$ is a compact subset of $\mathbb{R}^{2}$.

We write $R_{\theta}$ for the rotation around the $x_{3}$-axis by the angle $\theta$,

$$
R_{\theta}=\left(\begin{array}{ccc}
\cos \theta & \sin \theta & 0 \\
-\sin \theta & \cos \theta & 0 \\
0 & 0 & 1
\end{array}\right),
$$

and we denote by $S_{\theta}$ the superposition of $R_{\theta}$ and a translation along the $x_{3}$-direction of size $\kappa \theta$,

$$
S_{\theta}(x)=R_{\theta}(x)+\left(\begin{array}{l}
0 \\
0 \\
\kappa \theta
\end{array}\right) .
$$

Definition 2.1. A vector field $u: \mathbb{R}^{3} \rightarrow \mathbb{R}^{3}$ is said to be helical if and only if

$$
u\left(S_{\theta} x\right)=R_{\theta} u(x) \text { for all } \theta \in \mathbb{R} \text { and } x \in \mathbb{R}^{3} .
$$


Observe that, if a vector field $u$ is helical, then it is periodic in the $x_{3}$-direction with period $2 \kappa \pi$.

Definition 2.2. Set

$$
\xi=\xi(x) \equiv\left(x_{2},-x_{1}, \kappa\right) .
$$

Given a vector field $u: \mathbb{R}^{3} \rightarrow \mathbb{R}^{3}$, we define the helical swirl $\eta$ as

$$
\eta(x)=u(x) \cdot \xi(x) \text { for all } x \in \mathbb{R}^{3} .
$$

If $\eta \equiv 0$ then we say that $u$ has vanishing helical swirl.

Next, we state some properties of helical flows. The proofs can be found in [9].

Lemma 2.1. A smooth vector field $u: \mathbb{R}^{3} \rightarrow \mathbb{R}^{3}$ is helical if and only if $(\xi(x) \cdot \nabla) u(x)=$ $\left(u_{2}(x),-u_{1}(x), 0\right)$.

Lemma 2.2. Let $u=u(x, t)$ be a smooth solution of (1.1), helical and with vanishing helical swirl and let $\boldsymbol{\omega}=$ curl $u$. Then $\boldsymbol{\omega}(x, t)=\omega(x, t) \xi(x) / \kappa$, where $\omega(x, t)=\partial_{1} u_{2}(x, t)-\partial_{2} u_{1}(x, t)$, and $\boldsymbol{\omega}$ satisfies the following equation:

$$
\partial_{t} \boldsymbol{\omega}+(u \cdot \nabla) \boldsymbol{\omega}-\frac{\omega}{\kappa} \mathcal{R} u=0
$$

where $\mathcal{R}=\left(\begin{array}{rrr}0 & 1 & 0 \\ -1 & 0 & 0 \\ 0 & 0 & 0\end{array}\right)$.

Remark 2.1. The third equation in system (2.2) is a transport equation for $\omega$, given by

$$
\partial_{t} \omega+(u \cdot \nabla) \omega=0
$$

Observe that, if $u \in L^{q}\left(\mathbb{R}^{3} ; \mathbb{R}^{3}\right)$, for some $1 \leq q \leq \infty$, then Definitions (2.1) and (2.2) still make sense if we ask the equalities to hold for almost every $x \in \mathbb{R}^{3}$. Furthermore, in this case we have a result analogous to Lemma 2.1, which is stated below, and can be proved in the same fashion.

Lemma 2.3. Let $u \in L_{l o c}^{q}\left(\mathbb{R}^{3} ; \mathbb{R}^{3}\right)$ for some $1 \leq q \leq \infty$. Then, $u$ is helical if and only if $u$ is $2 \pi \kappa$-periodic with respect to the third component, in the sense of distributions, and if

$$
\int_{\Omega}(D \Psi(x) \xi(x)) \cdot u(x) d x=\int_{\Omega}(\mathcal{R} \Psi(x)) \cdot u(x) d x
$$

for all $\Psi \in \mathcal{C}_{c, p e r}^{\infty}\left(\Omega ; \mathbb{R}^{3}\right)$.

\section{VORTICITY FORMULATION}

In this section we state and prove some basic results concerning full-space helical flows which we will use in the proof of the existence theorems 4.1 and 5.1. We require an explicit vorticity formulation, and an appropriate Biot-Savart law. We begin with the following system

$$
\left\{\begin{array}{l}
\operatorname{curl} u=\omega \\
\operatorname{div} u=0 \\
|u(x)| \rightarrow 0 \text { as }|\tilde{x}| \rightarrow \infty \\
u \text { periodic in } x_{3} .
\end{array}\right.
$$

In order to obtain a solution for the system above we will derive an explicit form of the Green's function for the Laplacian in $\mathbb{R}^{3}$, periodic in the $x_{3}$-direction. In addition, we introduce a definition of weak solution for the vorticity equation (2.3) and, finally, we construct a smooth approximating sequence for weak solutions of the vorticity equation (2.3). 
3.1. Biot-Savart law. We will being by deriving the Green's function for the Laplacian in $\mathbb{R}^{3}$ with periodic boundary conditions in the $x_{3}$-direction. Using this Green's function we will write an expression for the Biot-Savart kernel and prove some estimates for this kernel. We will also provide a necessary and sufficient condition for a velocity field, associated with a given vorticity, to be helical and to have vanishing helical swirl (see Proposition 3.3).

Proposition 3.1. The Green's function for the Laplacian in $\mathbb{R}^{3}$ with $2 \pi \kappa$-periodic boundary condition in the $x_{3}$-direction is given by

$$
G(x)=\frac{1}{2 \pi \kappa^{2}} \sum_{n=1}^{\infty} K_{0}\left(\frac{|\tilde{x}| n}{\kappa}\right) \cos \left(\frac{n x_{3}}{\kappa}\right)-\frac{1}{2 \pi \kappa^{2}} \log |\tilde{x}|,
$$

for all $x \in \mathbb{R}^{3}, \tilde{x} \neq 0$, where $K_{0}$ is the modified Bessel function of the second kind and order zero.

In particular, if $\boldsymbol{\omega}: \mathbb{R}^{3} \rightarrow \mathbb{R}^{3}$ belongs to $L_{\text {per }}^{2}(\Omega)$ and if $\int_{\Omega} \boldsymbol{\omega}(x) d x=0$, then the solution of the Poisson system

$$
\left\{\begin{array}{l}
\Delta \Psi=\boldsymbol{\omega} \\
|\Psi(x)| \rightarrow 0 \text { as }|\tilde{x}| \rightarrow \infty \\
\Psi \text { periodic in } x_{3} .
\end{array}\right.
$$

is given by

$$
\Psi(x)=-\frac{1}{4 \pi^{2}} \int_{\Omega} G(x-y) \boldsymbol{\omega}(y) d y, \text { for all } x \in \Omega .
$$

This result can be proved using a standard approach by means of Fourier analysis. We omit the proof, and we refer the reader to [11].

Definition 3.1. Let $G$ be the Green's function given by (3.2). Set

$$
\mathcal{K}=\mathcal{K}(x):=\frac{1}{4 \pi^{2}} \nabla G(x), \text { for all } x \in\left(\mathbb{R}^{2} \backslash\{0\}\right) \times(-\pi \kappa, \pi \kappa) .
$$

We refer to the function $\mathcal{K}$ as the Biot-Savart kernel.

In the following result we obtain an estimate for the Biot-Savart kernel.

Lemma 3.1. The kernel $\mathcal{K}$, defined by (3.4), satisfies the following estimate

$$
|\mathcal{K}(x)| \leq C\left(\frac{1}{|x|^{2}}+\frac{1}{|\tilde{x}|}\right) \text {, for all } x \in\left(\mathbb{R}^{2} \backslash\{0\}\right) \times(-\pi \kappa, \pi \kappa) .
$$

Proof. Fix $x \in\left(\mathbb{R}^{2} \backslash\{0\}\right) \times(-\pi \kappa, \pi \kappa)$. First, we observe that

$$
|\mathcal{K}(x)| \leq \frac{1}{8 \pi^{3} \kappa^{2}}\left|\nabla\left(\sum_{n=1}^{\infty} K_{0}\left(\frac{|\tilde{x}| n}{\kappa}\right) \cos \left(\frac{n x_{3}}{\kappa}\right)\right)\right|+\frac{1}{8 \pi^{3} \kappa^{2}} \frac{1}{|\tilde{x}|} .
$$

In order to estimate the series involving the Bessel function we use the following expansion in Schloeminch series (see [15]),

$$
\begin{aligned}
\sum_{n=1}^{\infty} K_{0} & \left(\frac{|\tilde{x}|}{\kappa} n\right) \cos \left(\frac{x_{3}}{\kappa} n\right)=\frac{1}{2}\left(\ln \left(\frac{\gamma}{4 \pi \kappa}\right)+\ln |\tilde{x}|\right)+\frac{\pi \kappa}{2|x|}+ \\
+ & \frac{\pi \kappa}{2} \sum_{m=1}^{\infty}\left[\frac{1}{\sqrt{|\tilde{x}|^{2}+\left(2 \pi \kappa m-x_{3}\right)^{2}}}-\frac{1}{2 \pi \kappa m}\right]+ \\
+ & \frac{\pi \kappa}{2} \sum_{m=1}^{\infty}\left[\frac{1}{\sqrt{|\tilde{x}|^{2}+\left(2 \pi \kappa m+x_{3}\right)^{2}}}-\frac{1}{2 \pi \kappa m}\right] .
\end{aligned}
$$

To obtain the desired estimate (3.5) for the kernel $\mathcal{K}$ we need to estimate the gradient of the series in (3.6). It is not hard to see that the $m$-th term in each of the two series on the righthand-side of (3.6) is bounded by a multiple of $|x| / m\left(2 \pi \kappa m \pm x_{3}\right)$ and, hence, both series converge pointwise. Furthermore, for each of these two series we have that the derivative of the $m$-th term is 
bounded by $1 /(2 \pi \kappa(m-1 / 2))^{2}$, so that both series of derivatives are uniformly convergent. Thus, we can differentiate both series in (3.6) term by term and obtain that

$$
\begin{gathered}
\nabla\left(\sum_{n=1}^{\infty} K_{0}\left(\frac{|\tilde{x}|}{\kappa} n\right) \cos \left(\frac{x_{3}}{\kappa} n\right)\right) \\
=\frac{1}{2|\tilde{x}|^{2}}(\tilde{x}, 0)-\frac{\pi \kappa}{2|x|^{3}} x+\frac{\pi \kappa}{2}\left(\sum_{m=1}^{\infty} f_{m}^{-}(x)+\sum_{m=1}^{\infty} f_{m}^{+}(x)\right),
\end{gathered}
$$

where

$$
\left.f_{m}^{ \pm}(x)=\frac{1}{\left(|\tilde{x}|^{2}+\left(2 \pi \kappa m \pm x_{3}\right)^{2}\right)^{3 / 2}}\left(-x_{1},-x_{2}, \mp 2 \pi \kappa m-x_{3}\right)\right) .
$$

Next, let us estimate the series $\sum_{m=1}^{\infty} f_{m}^{-}$and $\sum_{m=1}^{\infty} f_{m}^{+}$. The idea is to compare the series with their corresponding integrals. In order to do so, define $h^{ \pm}(t)=\frac{1}{\left(|\tilde{x}|^{2}+\left(2 \pi \kappa t \pm x_{3}\right)^{2}\right)^{3 / 2}}$ and observe that $h^{ \pm}$is decreasing for all $t \geq 1$ and $h^{ \pm}(t) \geq h^{ \pm}(1)$, for all $0 \leq t \leq 1$. Thus, we can estimate each series by its corresponding integral as follows:

$$
\begin{gathered}
\sum_{m=1}^{\infty} \frac{1}{\left(|\tilde{x}|^{2}+\left(2 \pi \kappa m \pm x_{3}\right)^{2}\right)^{3 / 2}} \leq \\
\int_{0}^{\infty} \frac{1}{\left(|\tilde{x}|^{2}+\left(2 \pi \kappa t \pm x_{3}\right)^{2}\right)^{3 / 2}} d t=\frac{1}{2 \pi \kappa|\tilde{x}|^{2}} \frac{|x| \pm x_{3}}{|x|} \leq \frac{1}{\pi \kappa|\tilde{x}|^{2}} .
\end{gathered}
$$

We obtain the following estimate for the series involving each of the first two components of $f_{m}^{ \pm}$:

$$
\left|\sum_{m=1}^{\infty}\left(f_{m}^{ \pm}(x)\right)_{i}\right| \leq\left|x_{i}\right| \sum_{m=1}^{\infty} \frac{1}{\left(|\tilde{x}|^{2}+\left(2 \pi \kappa m \pm x_{3}\right)^{2}\right)^{3 / 2}} \leq \frac{\left|x_{i}\right|}{\pi \kappa|\tilde{x}|^{2}}, \quad \text { for } i=1,2 .
$$

The last component of $f_{m}^{ \pm}$is more delicate since the terms $\left(f_{m}^{ \pm}\right)_{3}$ do not have the same sign and do not form a decreasing sequence in $m$. Set $g^{ \pm}(t)=\frac{2 \pi \kappa t \pm x_{3}}{\left(|\tilde{x}|^{2}+\left(2 \pi \kappa t \pm x_{3}\right)^{2}\right)^{3 / 2}}$ and observe that $g^{ \pm}(t) \leq 0$ if $t \leq \mp x_{3} /(2 \pi \kappa)$ and $g^{ \pm}(t) \geq 0$ if $t \geq \mp x_{3} /(2 \pi \kappa)$. Furthermore,

$$
\left(g^{ \pm}\right)^{\prime}(t)=\frac{2 \pi \kappa\left(|\tilde{x}|^{2}-2\left(2 \pi \kappa t \pm x_{3}\right)^{2}\right)}{\left(|\tilde{x}|^{2}+\left(2 \pi \kappa t \pm x_{3}\right)^{2}\right)^{5 / 2}} .
$$

Thus, $\left(g^{ \pm}\right)^{\prime}(t) \leq 0$ if and only if $t \geq \frac{1}{2 \pi \kappa}\left(\frac{|\tilde{x}|}{\sqrt{2}} \mp x_{3}\right)$. We will divide our analysis in two cases. First we assume that $\frac{1}{2 \pi \kappa}\left(\frac{|\tilde{x}|}{\sqrt{2}} \mp x_{3}\right) \geq 1$ and we split the series as follows:

$$
\begin{gathered}
\sum_{m=1}^{\infty} \frac{2 \pi \kappa m \pm x_{3}}{\left(|\tilde{x}|^{2}+\left(2 \pi \kappa m \pm x_{3}\right)^{2}\right)^{3 / 2}}= \\
=\sum_{m=1}^{d} \frac{2 \pi \kappa m \pm x_{3}}{\left(|\tilde{x}|^{2}+\left(2 \pi \kappa m \pm x_{3}\right)^{2}\right)^{3 / 2}}+\sum_{m=d+1}^{\infty} \frac{2 \pi \kappa m \pm x_{3}}{\left(|\tilde{x}|^{2}+\left(2 \pi \kappa m \pm x_{3}\right)^{2}\right)^{3 / 2}}
\end{gathered}
$$

where $d=\left[\frac{1}{2 \pi \kappa}\left(\frac{|\tilde{x}|}{\sqrt{2}} \mp x_{3}\right)\right]$ (here, $[y]$ denotes the greatest integer less than y). Therefore we have:

$$
\begin{gathered}
\sum_{n=1}^{\infty} \frac{2 \pi \kappa n \pm x_{3}}{\left(|\tilde{x}|^{2}+\left(2 \pi \kappa n \pm x_{3}\right)^{2}\right)^{3 / 2}} \leq \\
\leq d \frac{2 \pi \kappa d \pm x_{3}}{\left(|\tilde{x}|^{2}+\left(2 \pi \kappa d \pm x_{3}\right)^{2}\right)^{3 / 2}}+\int_{d}^{\infty} \frac{2 \pi \kappa t \pm x_{3}}{\left(|\tilde{x}|^{2}+\left(2 \pi \kappa t \pm x_{3}\right)^{2}\right)^{3 / 2}} d t= \\
=d \frac{2 \pi \kappa d \pm x_{3}}{\left(|\tilde{x}|^{2}+\left(2 \pi \kappa d \pm x_{3}\right)^{2}\right)^{3 / 2}}+\frac{1}{2 \pi \kappa} \frac{1}{\sqrt{|\tilde{x}|^{2}+\left(2 \pi \kappa d \pm x_{3}\right)^{2}}} \leq \\
\leq \frac{1}{2 \pi \kappa}\left(\frac{|\tilde{x}|}{\sqrt{2}} \mp x_{3}\right) \frac{1}{\sqrt{2}|\tilde{x}|^{2}}+\frac{1}{2 \pi \kappa} \frac{1}{|\tilde{x}|} \leq
\end{gathered}
$$




$$
\leq \frac{1}{2 \pi \kappa}\left(\frac{2|\tilde{x}|}{\sqrt{2}}\right) \frac{1}{\sqrt{2}|\tilde{x}|^{2}}+\frac{1}{2 \pi \kappa} \frac{1}{|\tilde{x}|}=\frac{1}{\pi \kappa} \frac{1}{|\tilde{x}|} .
$$

For the last inequality observe that, since $\frac{1}{2 \pi \kappa}\left(\frac{|\tilde{x}|}{\sqrt{2}} \mp x_{3}\right) \geq 1$ we get $\frac{|\tilde{x}|}{\sqrt{2}} \mp x_{3} \leq \frac{2|\tilde{x}|}{\sqrt{2}}$. Hence,

$$
\sum_{n=1}^{\infty} \frac{2 \pi \kappa n \pm x_{3}}{\left(|\tilde{x}|^{2}+\left(2 \pi \kappa n \pm x_{3}\right)^{2}\right)^{3 / 2}} \leq \frac{1}{\pi \kappa} \frac{1}{|\tilde{x}|} .
$$

Next assume that $\frac{1}{2 \pi \kappa}\left(\frac{|\tilde{x}|}{\sqrt{2}} \mp x_{3}\right) \leq 1$. Then it is clear that

$$
\begin{gathered}
\sum_{m=1}^{\infty} \frac{2 \pi \kappa m \pm x_{3}}{\left(|\tilde{x}|^{2}+\left(2 \pi \kappa m \pm x_{3}\right)^{2}\right)^{3 / 2}} \leq \\
\leq \frac{2 \pi \kappa \pm x_{3}}{\left(|\tilde{x}|^{2}+\left(2 \pi \kappa \pm x_{3}\right)^{2}\right)^{3 / 2}}+\int_{1}^{\infty} \frac{2 \pi \kappa t \pm x_{3}}{\left(|\tilde{x}|^{2}+\left(2 \pi \kappa t \pm x_{3}\right)^{2}\right)^{3 / 2}} d t \\
=\frac{2 \pi \kappa \pm x_{3}}{\left(|\tilde{x}|^{2}+\left(2 \pi \kappa \pm x_{3}\right)^{2}\right)^{3 / 2}}+\frac{1}{2 \pi \kappa} \frac{1}{\sqrt{|\tilde{x}|^{2}+\left(2 \pi \kappa \pm x_{3}\right)^{2}}} \\
\leq \frac{1}{\sqrt{|\tilde{x}|^{2}+\left(2 \pi \kappa \pm x_{3}\right)^{2}}}\left(\frac{1}{2 \pi \kappa \pm x_{3}}+\frac{1}{2 \pi \kappa}\right) \leq \frac{3}{2 \pi \kappa|\tilde{x}|} .
\end{gathered}
$$

Therefore, combining the last two estimates, we obtain that

$$
\sum_{m=1}^{\infty}\left(f_{m}^{ \pm}(x)\right)_{3}=\sum_{n=1}^{\infty} \frac{2 \pi \kappa n \pm x_{3}}{\left(|\tilde{x}|^{2}+\left(2 \pi \kappa n \pm x_{3}\right)^{2}\right)^{3 / 2}} \leq \frac{3}{2 \pi \kappa|\tilde{x}|}
$$

Finally, combining (3.7) and (3.9) we find

$$
\left|\sum_{m=1}^{\infty} f_{m}^{-}(x)+\sum_{m=1}^{\infty} f_{m}^{+}(x)\right| \leq 2\left(\left|x_{1}\right|+\left|x_{2}\right|\right) \frac{1}{\pi \kappa} \frac{1}{|\tilde{x}|^{2}}+2 \frac{3}{2 \pi \kappa} \frac{1}{|\tilde{x}|} \leq \frac{5}{\pi \kappa} \frac{1}{|\tilde{x}|} .
$$

Consequently,

$$
|\mathcal{K}(x)| \leq C\left(\frac{1}{|x|^{2}}+\frac{1}{|\tilde{x}|}\right)
$$

Next, we provide a decay property for a convolution-type operator associated with the BiotSavart kernel.

Lemma 3.2. Let $\Phi \in \mathcal{C}_{c, p e r}^{\infty}\left(\Omega ; \mathbb{R}^{3}\right)$ be a vector field such that $\int_{\Omega} \Phi(x) d x=0$. Let $I=I(x)$ be given by

$$
I(x):=\int_{\Omega} \mathcal{K}(x-y) \times \Phi(y) d y, \text { for all } x \in \Omega .
$$

Then, $|I(x)|=\mathcal{O}\left(|\tilde{x}|^{-2}\right)$ as $|\tilde{x}| \rightarrow \infty$.

Proof. Let $R>0$ be such that supp $\Phi \subset B(0, R) \times \mathbb{R}$, where $B(0, R)$ is the ball in $\mathbb{R}^{2}$ centered at the origin, with radius $R$. Consider any $x \in \Omega$ such that $|\tilde{x}| \geq 2 R$. Using (3.4) and the fact that $K_{0}^{\prime}=-K_{1}$ we obtain that

$$
\begin{gathered}
\mathcal{K}(x)=\frac{1}{8 \pi^{3} \kappa^{2}} \nabla\left(\sum_{n=1}^{\infty} K_{0}\left(\frac{|\tilde{x}| n}{\kappa}\right) \cos \left(\frac{n x_{3}}{\kappa}\right)\right)-\frac{1}{8 \pi^{3} \kappa^{2}} \frac{1}{|\tilde{x}|^{2}}(\tilde{x}, 0) \\
=\frac{-1}{8 \pi^{3} \kappa^{2}}\left(\sum_{n=1}^{\infty}\left(K_{1}\left(\frac{|\tilde{x}| n}{\kappa}\right) \cos \left(\frac{n x_{3}}{\kappa}\right) \frac{n \tilde{x}}{\kappa|\tilde{x}|}, K_{0}\left(\frac{|\tilde{x}| n}{\kappa}\right) \sin \left(\frac{n x_{3}}{\kappa}\right) \frac{n}{\kappa}\right)\right) \\
-\frac{1}{8 \pi^{3} \kappa^{2}} \frac{1}{|\tilde{x}|^{2}}(\tilde{x}, 0)=\mathcal{K}_{1}(x)-\mathcal{K}_{2}(x) .
\end{gathered}
$$


To estimate $\mathcal{K}_{1}$ we recall that Bessel function of the second kind $K_{\nu}$ is positive and decreasing for all $\nu>0, \int_{0}^{\infty} t K_{0}(t) d t=1$ and $\int_{0}^{\infty} t K_{1}(t) d t=\pi / 2$. Thus,

$$
\begin{gathered}
\left|\mathcal{K}_{1}(x)\right| \leq \frac{1}{8 \pi^{3} \kappa^{2}} \sum_{n=1}^{\infty} \frac{n}{\kappa}\left(K_{1}\left(\frac{|\tilde{x}| n}{\kappa}\right)+K_{0}\left(\frac{|\tilde{x}| n}{\kappa}\right)\right) \\
\leq \frac{1}{8 \pi^{3} \kappa^{3}} \int_{0}^{\infty} t\left(K_{1}\left(\frac{|\tilde{x}| t}{\kappa}\right)+K_{0}\left(\frac{|\tilde{x}| t}{\kappa}\right)\right) d t= \\
=\frac{1}{8 \pi^{3} \kappa^{3}} \int_{0}^{\infty} \frac{s \kappa}{|\tilde{x}|}\left(K_{1}(s)+K_{0}(s)\right) \frac{\kappa}{|\tilde{x}|} d s \leq \frac{C}{|\tilde{x}|^{2}} .
\end{gathered}
$$

Thus,

$$
\left|\int_{\Omega} \mathcal{K}_{1}(x-y) \times \Phi(y) d y\right|=\mathcal{O}\left(|\tilde{x}|^{-2}\right) \text { as }|\tilde{x}| \rightarrow \infty .
$$

Next, we observe that, for $|\tilde{x}| \geq 2 R$ and $|\tilde{y}| \leq R$, we have that

$$
|\tilde{x}-\tilde{y}|^{-2}=|\tilde{x}|^{-2}+\mathcal{O}\left(|\tilde{x}|^{-3}\right)
$$

so that

$$
\begin{gathered}
\int_{\Omega} \mathcal{K}_{2}(x-y) \times \Phi(y) d y=\frac{1}{8 \pi^{3} \kappa^{2}} \int_{\Omega} \frac{1}{|\tilde{x}-\tilde{y}|^{2}}(\tilde{x}-\tilde{y}, 0) \times \Phi(y) d y \\
=\frac{1}{8 \pi^{3} \kappa^{2}} \frac{1}{|\tilde{x}|^{2}}(\tilde{x}, 0) \times \int_{\Omega} \Phi(y) d y+\mathcal{O}\left(|\tilde{x}|^{-2}\right)=\mathcal{O}\left(|\tilde{x}|^{-2}\right) .
\end{gathered}
$$

In view of the estimates obtained we find: $|I(x)|=\mathcal{O}\left(|\tilde{x}|^{-2}\right)$ for $|\tilde{x}| \geq 2 R$.

Corollary 3.1. Let $\Phi \in \mathcal{C}_{c, p e r}^{\infty}\left(\Omega ; \mathbb{R}^{3}\right)$ and assume that $\int_{\Omega} \Phi(x) d x=0$. Then the function I given by

$$
I=I(x):=\int_{\Omega} \mathcal{K}(x-y) \times \Phi(y) d y
$$

belongs to $L^{r}\left(\Omega ; \mathbb{R}^{3}\right)$, for any $r>1$.

Proof. Let $R>0$ be such that supp $\Phi \subset B(0, R) \times \mathbb{R}$. It follows from the proof of Lemma 3.2 that $|I(x)|=\mathcal{O}\left(|\tilde{x}|^{-2}\right)$ for $|\tilde{x}| \geq 2 R$. Thus, for all $r>1, u \in L^{r}\left(\Omega \cap\left(B(0,2 R)^{c} \times \mathbb{R}\right)\right)$. On the other hand, using that $\mathcal{K} \in L_{\text {loc }}^{s}(\Omega)$, for $1 \leq s<3 / 2, \Phi \in L^{p}(\Omega)$, for $1 \leq p \leq \infty$, and the Generalized Young inequality on $U=\Omega \cap(B(0,3 R) \times \mathbb{R})$, we obtain that

$$
\|\mathcal{K} * \Phi\|_{L^{r}(U)} \leq\|\mathcal{K}\|_{L^{s}(U)}\|\Phi\|_{L^{p}(U)}
$$

where $1 / r=1 / p+1 / s-1$. We conclude by observing that we can cover all $r$ in the interval $(1, \infty]$ by choosing, for instance, $s=1$ and $p=r$.

We are now ready to provide a characterization of the velocity field in terms of vorticity, i.e., the so called Biot-Savart law.

Proposition 3.2. Let $\Phi \in \mathcal{C}_{c, p e r}^{\infty}\left(\Omega ; \mathbb{R}^{3}\right)$ be a vector field such that div $\Phi=0$ and $\int_{\Omega} \Phi d x=0$.

Then there exists a unique smooth solution of

$$
\left\{\begin{array}{l}
\text { curl } u=\Phi \\
\text { div } u=0 \\
|u(x)| \rightarrow 0 \text { as }|\tilde{x}| \rightarrow \infty \\
u \text { periodic in } x_{3}
\end{array}\right.
$$

and it is given by

$$
u=u(x):=\int_{\Omega} \mathcal{K}(x-y) \times \Phi(y) d y
$$


Proof. We start by proving that the vector field given by (3.12) is indeed a solution of (3.11). Since $\Phi \in \mathcal{C}_{c, p e r}^{\infty}\left(\Omega ; \mathbb{R}^{3}\right)$, it is clear that $u \in \mathcal{C}_{p e r}^{\infty}\left(\Omega ; \mathbb{R}^{3}\right)$, i.e., $u$ is smooth and periodic in $x_{3}$. Moreover, using Lemma 3.2 we have that $u(x)=O\left(|\tilde{x}|^{-2}\right)$, and, hence, $|u(x)| \rightarrow 0$ as $|\tilde{x}| \rightarrow \infty$. Next, recall that $\mathcal{K}(x)=1 /\left(4 \pi^{2}\right) \nabla G(x)$, for all $x \in \Omega, \tilde{x} \neq 0$, where $G$ is given by (3.2). Since $\Phi \in \mathcal{C}_{c, p e r}^{\infty}\left(\Omega ; \mathbb{R}^{3}\right)$ a straightforward calculation yields

$$
u(x)=-\operatorname{curl}\left(-\frac{1}{4 \pi^{2}} \int_{\Omega} G(x-y) \Phi(y) d y\right) .
$$

Hence, div $u=0$. Define $\Psi(x)=-1 /\left(4 \pi^{2}\right) \int_{\Omega} G(x-y) \Phi(y) d y$, for all $x \in \Omega$, and observe that, by Proposition 3.1 $\Delta \Psi=\Phi$. Thus, using that $-\operatorname{curl}$ curl $\Psi=\Delta \Psi-\nabla \operatorname{div} \Psi$, we obtain

$$
\text { curl } u=\Phi-\nabla \operatorname{div} \Psi \text {. }
$$

To conclude the proof that $u$ is a solution of (3.11) it remains only to show that $\nabla \operatorname{div} \Psi=0$. To see this, introduce the vector fields $f:=\nabla \operatorname{div} \Psi$ and $g:=\operatorname{curl} u$. Since $\Psi, \Phi \in \mathcal{C}_{p e r}^{\infty}\left(\Omega ; \mathbb{R}^{3}\right)$, we obtain the following identities

$$
\begin{gathered}
f(x)=-\int_{\Omega} \mathcal{K}(x-y) \operatorname{div} \Phi(y) d y \\
g(x)=\int_{\Omega} \mathcal{K}(x-y) \times \operatorname{curl} \Phi(y) d y,
\end{gathered}
$$

so that, by Lemma 3.2, we have that $f=\mathcal{O}\left(|\tilde{x}|^{-2}\right)$ and $g=\mathcal{O}\left(|\tilde{x}|^{-2}\right)$. Therefore, $f$ and $g$ belong to $L^{2}(\Omega)$. Taking the $L^{2}$-inner product between $f$ and identity (3.13) we obtain that

$$
(f, f)_{2}=(\Phi, f)_{2}-(g, f)_{2} \text {. }
$$

We have that $f$ is the gradient of $\operatorname{div} \Psi$, $\operatorname{div} g=0$ and, by hypothesis, $\operatorname{div} \Phi=0$. Thus, we have that $(\Phi, f)_{2}=(g, f)_{2}=0$. Therefore, $(f, f)_{2}=0$, which, in turn, implies that $\nabla \operatorname{div} \Psi \equiv 0$.

Finally, let us prove that the system (3.11) has a unique solution. Suppose that we have two solutions of (3.11), namely $u_{1}$ and $u_{2}$. Of course, $U:=u_{1}-u_{2}$ is a solution of (3.11), with $\Phi=0$, that is,

$$
\left\{\begin{array}{l}
\operatorname{curl} U=0 \\
\operatorname{div} U=0 \\
|U(x)| \rightarrow 0 \text { as }|\tilde{x}| \rightarrow \infty \\
U \text { periodic in } x_{3} .
\end{array}\right.
$$

Using the identity curl curl $U=-\Delta U+\nabla \operatorname{div} U$ and the fact that $U$ is a solution of (3.14) it follows that $\Delta U=0$. Observe that $U$ can be regarded as a harmonic function in the full-space, which is $2 \pi \kappa$-periodic with respect to the third component, and which decays to zero as $|\tilde{x}|$ goes to infinity, so that $U$ is bounded in $\mathbb{R}^{3}$. It follows from Liouville's theorem (see e.g. [10]) that $U \equiv 0$.

3.2. Weak solution. We begin by giving the definition of a weak solution; this formulation is inspired on Schochet's weak vorticity formulation for the $2 D$ incompressible Euler equations, see [20].

Definition 3.2. Let $\omega^{0} \in L_{c, p e r}^{p}(\Omega ; \mathbb{R})$ for some $p>4 / 3$. We say that a scalar function $\omega=\omega(t, x)$ is a weak solution of

$$
\partial_{t} \omega+(u \cdot \nabla) \omega=0
$$

in the time interval $(0, T)$ with initial data $\omega^{0}$, if

(i) $\omega \in L^{\infty}\left(0, T ; L_{p e r}^{p}(\Omega ; \mathbb{R})\right)$,

(ii) the associated velocity field

$$
u(t, x)=\int_{\Omega} \mathcal{K}(x-y) \times \frac{\xi(y)}{\kappa} \omega(t, y) d y
$$

belongs to $L^{\infty}\left(0, T ; L_{\text {per }}^{2}\left(\Omega ; \mathbb{R}^{3}\right)\right)$,

(iii) for all test functions $\psi \in \mathcal{C}_{c, p e r}^{\infty}([0, T) \times \Omega ; \mathbb{R})$ the following identity holds true: 


$$
\begin{gathered}
\int_{0}^{T} \int_{\Omega} \psi_{t}(t, x) \omega(t, x) d x d t+\int_{0}^{T} \int_{\Omega} \int_{\Omega} \mathcal{H}_{\psi}(t, x, y) \omega(t, y) \omega(t, x) d y d x d t \\
+\int_{\Omega} \psi(x, 0) \omega^{0}(x) d x=0,
\end{gathered}
$$

where

$$
\mathcal{H}_{\psi}(t, x, y)=\frac{1}{2 \kappa} \mathcal{K}(x-y) \cdot(\xi(y) \times(\nabla \psi(t, x)-\nabla \psi(t, y))-(\xi(x)-\xi(y)) \times \nabla \psi(t, y)) .
$$

Remark 3.1. We note in passing that the integrals in Definition 3.2 are convergent. Indeed, using Hölder's inequality we have that

$$
\begin{array}{r}
\quad\left|\int_{0}^{T} \int_{\Omega} \psi_{t}(t, x) \omega(t, x) d x d t+\int_{\Omega} \psi(x, 0) \omega^{0}(x) d x\right| \\
\leq\left\|\psi_{t}\right\|_{L^{1}\left(L^{q}\right)}\|\omega\|_{L^{\infty}\left(L^{p}\right)}+\|\psi(0, \cdot)\|_{L^{q}}\left\|\omega^{0}\right\|_{L^{p}}<\infty,
\end{array}
$$

where $1 / p+1 / q=1$. It remains to prove that the following integral is bounded:

$$
\int_{0}^{T} \int_{\Omega} \int_{\Omega} \mathcal{H}_{\psi}(t, x, y) \omega(t, y) \omega(t, x) d y d x d t
$$

To see that this integral is finite we divide the domain $\Omega \times \Omega \times(0, T)$ into subdomains as follows. Fix $\delta>0$ and $R>0$ and let $R_{1}=\{(x, y) \in \Omega \times \Omega:|x-y|<\delta\}, R_{2}=\{(x, y) \in \Omega \times \Omega:|x-y| \geq$ $\delta,|x|<R\}$ and $R_{3}=\{(x, y) \in \Omega \times \Omega:|x-y| \geq \delta,|x| \geq R\}$. Performing estimates in each region yields the desired result; the proof follows the same steps as in the proof of Theorem 4.1, so we omit the details here.

Our next proposition consists in basic properties relating the velocity field and the corresponding vorticity.

Proposition 3.3. Let $\boldsymbol{\omega} \in L_{c, p e r}^{p}\left(\Omega ; \mathbb{R}^{3}\right)$ and let $u(x)=(\mathcal{K} * \boldsymbol{\omega})(x)$, for all $x \in \Omega$. Then, $u$ is helical and has vanishing helical swirl if and only if $\boldsymbol{\omega}=\left(\partial_{1} u_{2}-\partial_{2} u_{1}\right) \xi / \kappa$ in $\mathcal{D}^{\prime}\left(\Omega ; \mathbb{R}^{3}\right)$.

Proof. First observe that, since $\boldsymbol{\omega}=$ curl $u$ in $\mathcal{D}^{\prime}$, for all $\Psi \in \mathcal{C}_{c}^{\infty}\left(\Omega ; \mathbb{R}^{3}\right)$ we have that $\langle\boldsymbol{\omega}, \Psi\rangle=$ $\langle\operatorname{curl} u, \Psi\rangle=\langle u$, curl $\Psi\rangle$. Suppose that $u$ is helical and has vanishing swirl, that is, $\eta=u \cdot \xi=0$, then $u_{3}(x)=\left(-x_{2} u_{1}(x)+x_{1} u_{2}(x)\right) / \kappa$. Using this fact and Lemma 2.3 we obtain the following

$$
\begin{gathered}
\langle\boldsymbol{\omega}, \Psi\rangle=\frac{1}{\kappa}\left\langle\left(\begin{array}{c}
\kappa u_{1} \\
\kappa u_{2} \\
-x_{2} u_{1}+x_{1} u_{2}
\end{array}\right), \operatorname{curl} \Psi\right\rangle= \\
=\frac{1}{\kappa}\left\langle\operatorname{curl}\left(\begin{array}{c}
\kappa u_{1} \\
\kappa u_{2} \\
-x_{2} u_{1}+x_{1} u_{2}
\end{array}\right), \Psi\right\rangle \\
=\frac{1}{\kappa}\langle\omega \xi, \Psi\rangle+\frac{1}{\kappa}\left\langle\left(\begin{array}{c}
-\xi \cdot \nabla u_{2}-u_{1} \\
\xi \cdot \nabla u_{1}-u_{2} \\
0
\end{array}\right), \Psi\right\rangle \\
=\frac{1}{\kappa}\langle\omega \xi, \Psi\rangle+\frac{1}{\kappa}\left\langle\left(\begin{array}{c}
u_{1} \\
u_{2} \\
u_{3}
\end{array}\right),(\xi \cdot \nabla)\left(\begin{array}{c}
-\Psi_{2} \\
\Psi_{1} \\
0
\end{array}\right)\right\rangle-\frac{1}{\kappa}\left\langle\left(\begin{array}{c}
u_{1} \\
u_{2} \\
u_{3}
\end{array}\right), \mathcal{R}\left(\begin{array}{c}
-\Psi_{2} \\
\Psi_{1} \\
0
\end{array}\right)\right\rangle= \\
=\frac{1}{\kappa}\langle\omega \xi, \Psi\rangle .
\end{gathered}
$$

Therefore, for all $\Psi \in \mathcal{C}_{c}^{\infty}\left(\Omega ; \mathbb{R}^{3}\right)$ we have that $\langle\boldsymbol{\omega}, \Psi\rangle=\langle\omega \xi / \kappa, \Psi\rangle$.

Conversely, suppose that $\boldsymbol{\omega}=\omega \xi / \kappa$, where $\omega=\partial_{1} u_{2}-\partial_{2} u_{1}$. First observe that (curl $\left.\boldsymbol{\omega}\right) \cdot \xi=$ $-2 \omega$. Indeed, for all $\varphi \in \mathcal{C}_{c}^{\infty}(\Omega ; \mathbb{R})$ we have that

$$
\begin{gathered}
\langle(\operatorname{curl} \boldsymbol{\omega}) \cdot \xi, \varphi\rangle=\langle\operatorname{curl} \boldsymbol{\omega}, \xi \varphi\rangle=\langle\boldsymbol{\omega}, \operatorname{curl}(\xi \varphi)\rangle=\langle\boldsymbol{\omega}, \varphi \operatorname{curl} \xi+\nabla \varphi \times \xi\rangle \\
\left.=\left\langle\boldsymbol{\omega},-2 \varphi e_{3}+\nabla \varphi \times \xi\right\rangle=\underset{9}{\langle} \frac{1}{\kappa} \omega \xi,-2 \varphi e_{3}+\nabla \varphi \times \xi\right\rangle=
\end{gathered}
$$




$$
=\left\langle\frac{1}{\kappa} \omega, \xi \cdot\left(-2 \varphi e_{3}+\nabla \varphi \times \xi\right)\right\rangle=\langle-2 \omega, \varphi\rangle .
$$

Since $\boldsymbol{\omega}=$ curl $u$ and $\operatorname{div} u=0$ in $\mathcal{D}^{\prime}$ we also have that curl $\boldsymbol{\omega}=-\Delta u$ in $\mathcal{D}^{\prime}$. Furthermore, since $\Delta(u \cdot \xi)=\Delta u \cdot \xi+u \cdot \Delta \xi+2 \sum_{i=1}^{3} \nabla u_{i} \cdot \nabla \xi_{i}$ and $\Delta \xi=0$ we obtain that

$$
\Delta(u \cdot \xi)=-\operatorname{curl} \boldsymbol{\omega} \cdot \xi+2 \sum_{i=1}^{3} \nabla u_{i} \cdot \nabla \xi_{i}=2 \omega-2 \omega=0
$$

in $\mathcal{D}^{\prime}$. Thus, using Lemma 3.2, $u \cdot \xi$ solves the following equation in the sense of distributions:

$$
\left\{\begin{array}{l}
\Delta(u \cdot \xi)=0 \text { in } \Omega \\
|u(x) \cdot \xi(x)| \rightarrow 0 \text { as }|\tilde{x}| \rightarrow \infty
\end{array}\right.
$$

Therefore, $u \cdot \xi=0$, i.e., $u$ has vanishing helical swirl.

Finally we prove that $u$ is helical. To do so, we note that, in view of Lemma 2.3, it is enough to establish that, for all $\Psi \in \mathcal{C}_{c}^{\infty}\left(\Omega ; \mathbb{R}^{3}\right)$,

$$
\int_{\Omega} u(x) \cdot((\xi(x) \cdot \nabla) \Psi(x)) d x=\int_{\Omega} u(x) \cdot(\mathcal{R} \Psi(x)) d x .
$$

To prove (3.17) we begin by showing that, for all $\Psi \in \mathcal{C}_{c}^{\infty}\left(\Omega ; \mathbb{R}^{3}\right)$,

$$
\int_{\Omega} u_{3}(x)(\xi(x) \cdot \nabla) \Psi_{3}(x) d x=0 .
$$

Indeed, since curl $u=\omega=\omega \xi / \kappa$, we have

$$
\left\langle u, \operatorname{curl}\left(\left(x_{1}, x_{2}, 0\right) \Psi_{3}\right)\right\rangle=\left\langle\operatorname{curl} u,\left(x_{1}, x_{2}, 0\right) \Psi_{3}\right\rangle=\left\langle\boldsymbol{\omega} \cdot\left(x_{1}, x_{2}, 0\right), \Psi_{3}\right\rangle=0 .
$$

As $u \cdot \xi=0$ a.e., we also have that

$$
\begin{gathered}
\left\langle u, \operatorname{curl}\left(\left(x_{1}, x_{2}, 0\right) \Psi_{3}\right)\right\rangle=\left\langle u,\left(\begin{array}{c}
-x_{2} \partial_{3} \Psi_{3} \\
x_{1} \partial_{3} \Psi_{3} \\
x_{2} \partial_{1} \Psi_{3}-x_{1} \partial_{2} \Psi_{3}
\end{array}\right)\right\rangle \\
=\left\langle-x_{2} u_{1}+x_{1} u_{2}, \partial_{3} \Psi_{3}\right\rangle+\left\langle u_{3}, x_{2} \partial_{1} \Psi_{3}-x_{1} \partial_{2} \Psi_{3}\right\rangle \\
=\left\langle\kappa u_{3}, \partial_{3} \Psi_{3}\right\rangle+\left\langle u_{3}, x_{2} \partial_{1} \Psi_{3}-x_{1} \partial_{2} \Psi_{3}\right\rangle=\left\langle u_{3},(\xi \cdot \nabla) \Psi_{3}\right\rangle .
\end{gathered}
$$

Therefore, $\left\langle u_{3},(\xi \cdot \nabla) \Psi_{3}\right\rangle=0$, which proves (3.18).

To conclude the proof that $u$ is helical it remains to prove that the right-hand-side of (3.17) also vanishes. To this end it is enough to show that

$$
\left\langle u_{1},\left(\xi \cdot \nabla \Psi_{1}\right)\right\rangle+\left\langle u_{2},\left(\xi \cdot \nabla \Psi_{2}\right)\right\rangle=\left\langle u_{1}, \Psi_{2}\right\rangle+\left\langle u_{2},-\Psi_{1}\right\rangle .
$$

Let $\Phi \in \mathcal{C}_{c}^{\infty}\left(\Omega ; \mathbb{R}^{3}\right)$ and use that $\boldsymbol{\omega}=\left(\partial_{1} u_{2}-\partial_{2} u_{1}\right) \xi / \kappa$ in $\mathcal{D}^{\prime}$ to obtain

$$
\begin{aligned}
\langle\boldsymbol{\omega}, \Phi\rangle & =\frac{1}{\kappa}\left\langle\left(\partial_{1} u_{2}-\partial_{2} u_{1}\right) \xi, \Phi\right\rangle=-\frac{1}{\kappa}\left(\left\langle u_{2}, \xi \cdot \partial_{1} \Phi-\Phi_{2}\right\rangle-\left\langle u_{1}, \xi \cdot \partial_{2} \Phi+\Phi_{1}\right\rangle\right) \\
& =-\frac{1}{\kappa}\left(-\left\langle u_{1}, \Phi_{1}\right\rangle-\left\langle u_{2}, \Phi_{2}\right\rangle-\left\langle u_{1}, \xi \cdot \partial_{2} \Phi\right\rangle+\left\langle u_{2}, \xi \cdot \partial_{1} \Phi\right\rangle\right) .
\end{aligned}
$$

Next, we observe that since $\boldsymbol{\omega}=\operatorname{curl} u$ and $u \cdot \xi=0$ in $\mathcal{D}^{\prime}$, we also get that

$$
\langle\boldsymbol{\omega}, \Phi\rangle=\langle u, \operatorname{curl} \Phi\rangle=\frac{1}{\kappa}\left\langle\left(\kappa u_{1}, \kappa u_{2},-x_{2} u_{1}+x_{1} u_{2}\right), \operatorname{curl} \Phi\right\rangle .
$$

Now, combining (3.20) and (3.21) we obtain that

$$
\left\langle u_{1},\left(\xi \cdot \nabla\left(-\Phi_{2}\right)\right)\right\rangle+\left\langle u_{2},\left(\xi \cdot \nabla \Phi_{1}\right)\right\rangle=\left\langle u_{1}, \Phi_{1}\right\rangle+\left\langle u_{2}, \Phi_{2}\right\rangle .
$$

Choosing $\Phi=\left(\Psi_{2},-\Psi_{1}, 0\right)$ in the last expression yields (3.19). Finally, combining (3.19) and (3.18) we obtain (3.17). 
3.3. Smooth solutions. Our next results consists in local well-posedness of the 3D Euler equations with helical symmetry and vanishing helical swirl in the full space for smooth initial data.

Recall the notation used in 18 where $V^{k}(\Omega)$ stands for the set of all vector-valued functions in the Sobolev space $H^{k}(\Omega)$ which are divergence free.

Theorem 3.1. For any initial velocity $u^{0} \in V^{k}(\Omega)$, with $k \geq 3$, there exists $T_{0}=T_{0}\left(\left\|u^{0}\right\|_{H^{k}}\right)>0$ such that, for any $T<T_{0}$ there exists a unique solution $u \in \mathcal{C}\left([0, T] ; V^{k}(\Omega)\right) \cap \mathcal{C}^{1}\left([0, T] ; V^{k-1}(\Omega)\right)$ of the $3 D$ Euler equations. Moreover, if $u^{0}$ is helical and has vanishing helical swirl, then $u(\cdot, t)$ also is helical and has vanishing helical swirl, for any fixed time $t \in[0, T]$.

The first part of the assertion of the previous theorem can be proved in the same fashion as the classical result in $\mathbb{R}^{3}$. The second part can be prove using Dutrifoy's argument (see $[\underline{8}$ ). We omit the proof.

Next, we will construct a sequence of smooth solutions, using Theorem 3.1 above, which is uniformly bounded in a suitable function space.

Theorem 3.2. Let $\omega^{0} \in L_{c, p e r}^{p}(\Omega ; \mathbb{R})$, for some $1<p \leq \infty$. For each $n \in \mathbb{N}$, define $\omega_{n}^{0}:=\rho_{n} * \omega^{0}$, where $\rho_{n}$ is the standard mollifier in $\mathbb{R}^{3}$ which is periodic with respect to the third component. Then,

(i) for each $n \in \mathbb{N}$, there exists a smooth solution $\omega_{n} \in L^{\infty}\left(0, \infty ; L_{c, p e r}^{p}(\Omega ; \mathbb{R})\right)$ of (3.15) with initial data $\omega_{n}^{0}$;

(ii) $\left\{\omega_{n}\right\}_{n}$ is uniformly bounded in $L^{\infty}\left(0, \infty ; L_{\text {per }}^{q}(\Omega ; \mathbb{R})\right)$, for all $1 \leq q \leq p$;

(iii) $\left\{\omega_{n}\right\}_{n}$ possesses a subsequence which converges weak-* in $L^{\infty}\left(0, \infty ; L_{p e r}^{q}(\Omega ; \mathbb{R})\right)$ to a limit $\omega$, for all $1<q \leq p$

(iv) $\|\omega(t, \cdot)\|_{L^{1}(\Omega)} \leq C$, for some constant $C>0$.

Proof. For each $n \in \mathbb{N}$ define $u_{n}^{0}(x)=\int_{\Omega} \mathcal{K}(x-y) \times(1 / \kappa) \xi(y) \omega_{n}^{0}(y) d y$. Then $u_{n}^{0} \in V^{k}$ (see Corollary 3.1) for all $k \in \mathbb{N}$, so we can use Theorem 3.1 to obtain, for each $n$, a time $T^{n}>0$ such that, for any $T<T^{n}$ there exists a unique solution $u_{n} \in \mathcal{C}^{1}\left([0, T] ; V^{k-1}\right)$ of the Euler equations. Moreover, for each $n, u_{n}$ is helical and has vanishing helical swirl. Thus, for each $n, \boldsymbol{\omega}_{n}=\operatorname{curl} u_{n}$ solves (2.2) and, therefore $\omega_{n}$ solves (2.3), whence $\omega_{n}\left(t, X_{n}(\alpha, t)\right)=\omega_{n}^{0}(\alpha)$, where $X_{n}(\alpha, t)$ is the particle trajectory, starting at $\alpha \in \Omega$, such that

$$
\left\{\begin{array}{l}
\frac{d X_{n}(t, \alpha)}{d t}=u_{n}\left(t, X_{n}(t, \alpha)\right) \\
\left.X_{n}(\alpha, t)\right|_{t=0}=\alpha
\end{array}\right.
$$

It follows that

$$
\int_{\Omega}\left|\omega_{n}(t, x)\right|^{p} d x=\int_{\Omega}\left|\omega_{n}^{0}(\alpha)\right|^{p} d \alpha, \text { for all } 1 \leq p<\infty, \text { and }\left\|\omega_{n}(t, \cdot)\right\|_{L^{\infty}(\Omega)}=\left\|\omega_{n}^{0}\right\|_{L^{\infty}(\Omega)} .
$$

Note that, since $\omega_{0} \in L_{c, p e r}^{p}(\Omega)$, we have $\omega_{0} \in L_{\text {per }}^{q}(\Omega)$ for all $1 \leq q \leq p$. Therefore,

$$
\left\|\omega_{n}(t, \cdot)\right\|_{L^{q}(\Omega)}=\left\|\omega_{n}^{0}\right\|_{L^{q}(\Omega)} \leq\left\|\omega^{0}\right\|_{L^{q}(\Omega)} \leq C, \text { for all } 1 \leq q \leq p,
$$

which implies that $\left\{\omega_{n}\right\}_{n}$ is uniformly bounded in $L^{\infty}\left(0, \infty ; L_{p e r}^{q}(\Omega ; \mathbb{R})\right)$, for all $1 \leq q \leq p$.

Observe also that $\left\|\omega_{n}^{0}\right\|_{L^{\infty}(\Omega)} \leq C(n)\left\|\omega^{0}\right\|_{L^{p}(\Omega)}$, hence $\left\|\omega_{n}(t, \cdot)\right\|_{L^{\infty}(\Omega)} \leq C(n)$. Now, since $\boldsymbol{\omega}_{n}(t, x)=\omega_{n}(t, x) \xi(x) / \kappa$ we obtain that

$$
\left\|\boldsymbol{\omega}_{n}(t, \cdot)\right\|_{L^{\infty}}=\frac{1}{\kappa} \sup _{\alpha \in \operatorname{supp} w_{n}^{0}}\left|\omega_{n}^{0}(\alpha) \xi\left(X_{n}(t, \alpha)\right)\right| \leq C\left\|\omega_{n}^{0}\right\|_{L^{\infty}}\left(\kappa+\sup _{\alpha \in \operatorname{supp} \omega_{n}^{0}}\left|X_{n}(t, \alpha)\right|\right) .
$$

It remains to estimate $\sup _{\alpha \in \operatorname{supp} \omega_{n}^{0}}\left|X_{n}(t, \alpha)\right|$. To do so, define $R_{n}(t)=\sup _{\alpha \in K_{n}}\left|X_{n}(t, \alpha)\right|$ and $K_{n}=\operatorname{supp} \omega_{n}^{0}$. Observe that for all $\alpha^{\prime} \in K_{n}$ we have

$$
\begin{aligned}
& \frac{d}{d t}\left|X_{n}\left(t, \alpha^{\prime}\right)\right| \leq \sup _{\alpha \in K_{n}} \frac{d}{d t}\left|X_{n}(t, \alpha)\right| \leq \sup _{\alpha \in K_{n}}\left|\frac{d}{d t} X_{n}(t, \alpha)\right| \\
\leq & \sup _{\alpha \in K_{n}} \int_{\left|X_{n}(t, \alpha)-y\right| \leq 2 R_{n}(t)}\left|\mathcal{K}\left(X_{n}(t, \alpha)-y\right)\right||\xi(y)|\left|\omega_{n}(y)\right| d y
\end{aligned}
$$




$$
\begin{gathered}
\leq \sup _{\alpha \in K_{n}} \int_{\left|X_{n}(t, \alpha)-y\right| \leq 4 \kappa \pi}\left(\frac{1}{\left|X_{n}(t, \alpha)-y\right|^{2}}+\frac{1}{\left.\mid \tilde{X}_{n}(t, \alpha)-\tilde{y}\right) \mid}\right)\left(\left|\tilde{y}-\tilde{X}_{n}(t, \alpha)\right|+\left|\xi\left(X_{n}(t, \alpha)\right)\right|\right)\left|\omega_{n}(y)\right| d y \\
\quad+C \sup _{\alpha \in K_{n}} \int_{4 \kappa \pi \leq\left|X_{n}(t, \alpha)-y\right| \leq 2 R_{n}(t)}|\xi(y)|\left|\omega_{n}(y)\right| d y \\
\leq\left\|\omega_{n}\right\|_{L^{\infty}} \sup _{\alpha \in K_{n}} \int_{\left|X_{n}(t, \alpha)-y\right| \leq 4 \kappa \pi} \quad \begin{array}{c}
\left(\frac{1}{\left.\mid X_{n}(t, \alpha)-y\right) \mid}+1+\frac{\xi\left(X_{n}(t, \alpha)\right)}{\left.\mid X_{n}(t, \alpha)-y\right)\left.\right|^{2}}+\frac{\xi\left(X_{n}(t, \alpha)\right)}{\left.\mid \tilde{X}_{n}(t, \alpha)-\tilde{y}\right) \mid}\right) d y \\
+C R_{n}(t)\left\|\omega_{n}\right\|_{L^{1}} \leq C\left(\left\|\omega_{n}\right\|_{L^{\infty}}+\left\|\omega_{n}\right\|_{L^{1}}\right)\left(R_{n}(t)+1\right) \leq C(n)\left(R_{n}(t)+1\right),
\end{array}
\end{gathered}
$$

where we used Lemma 3.1 to estimate the kernel $\mathcal{K}$.

Hence,

$$
\frac{d}{d t}\left|X_{n}\left(t, \alpha^{\prime}\right)\right| \leq C(n)\left(R_{n}(t)+1\right)
$$

which implies, $\left|X_{n}\left(t, \alpha^{\prime}\right)\right| \leq\left|X_{n}\left(0, \alpha^{\prime}\right)\right|+C(n) \int_{0}^{t}\left(R_{n}(s)+1\right) d s$.

Taking the supremum in $\alpha^{\prime} \in K_{n}$ of the last identity we obtain

$$
R_{n}(t) \leq C+C(n) \int_{0}^{t}\left(R_{n}(s)+1\right) d s
$$

By Gronwall's lemma we have that $R_{n}(t) \leq(C+C(n) t) e^{C(n) t}$. Thus, $\left\|\boldsymbol{\omega}_{n}(t, \cdot)\right\|_{L^{\infty}} \leq(C+$ $C(n) t)\left\|\omega_{0}^{n}\right\|_{L^{\infty}} e^{C(n) t}$.

We have, hence,

$$
\int_{0}^{t}\left\|\boldsymbol{\omega}_{n}(s, \cdot)\right\|_{L^{\infty}} d s \leq\left\|\omega_{0}^{n}\right\|_{L^{\infty}} \int_{0}^{t}(C+C(n) s) e^{C(n) s} d s \leq\left\|\omega_{0}^{n}\right\|_{L^{\infty}}\left(C t+C(n) t^{2}\right) e^{C(n) t} .
$$

Therefore, by the Beale-Kato-Majda theorem we conclude that, for each $n \in \mathbb{N}, u_{n}$ can be continued, within its class, for all $T>0$. Therefore, $\omega_{n}$ is a global smooth solution of (3.15), for each $n \in \mathbb{N}$.

Since $\left\{\omega_{n}\right\}$ is uniformly bounded in $L^{\infty}\left(0, \infty ; L^{q}(\Omega)\right)$, for all $1 \leq q \leq p$, then there exists a subsequence, that we still denote by $\left\{\omega_{n}\right\}$, that converges weak-star in $L^{\infty}\left(0, \infty ; L^{q}(\Omega)\right)$ to a limit $\omega$, for all $1<q \leq p$. Since the total variation of a measure is weak-star lower semicontinuous and as $\left\|\omega_{n}(t, \cdot)\right\|_{L^{1}(\Omega)} \leq C$, we find that $\|\omega(t, \cdot)\|_{L^{1}(\Omega)} \leq C$.

\section{First existence theorem: Balanced vorticity}

In this section we prove existence of a weak solution to the helical vorticity equation for initial data with vanishing integral. This condition is needed since we are going to make use of the Biot-Savart law (3.12), which requires this hypothesis.

Theorem 4.1. Let $\omega_{0} \in L_{c, p e r}^{p}(\Omega ; \mathbb{R})$, for some $p>4 / 3$, such that $\int_{\Omega} \omega_{0}(x) d x=0$. Then, there exists a weak solution $\omega=\omega(t, x)$ of (3.15), in the sense of Definition [3.2, with initial data $\omega_{0}$.

Proof. We use Theorem 3.2 to obtain a sequence of smooth solutions $\left\{\omega_{n}\right\}$ of the vorticity equation satisfying the following properties

(i) $\omega_{n} \in L^{\infty}\left(0, \infty ; L_{c, p e r}^{p}(\Omega ; \mathbb{R})\right)$, for all $n$;

(ii) $\left\{\omega_{n}\right\}_{n=1}^{\infty}$ is uniformly bounded in $L^{\infty}\left(0, \infty, L_{p e r}^{q}(\Omega ; \mathbb{R})\right)$, for all $1 \leq q \leq p$;

(iii) the velocity field $u_{n}(x):=\int_{\Omega} \mathcal{K}(x-y) \times(\xi(y) / \kappa) \omega_{n}(t, y) d y$ is helical and has vanishing helical swirl for all $n$, and $\left\{u_{n}\right\}_{n=1}^{\infty}$ is uniformly bounded in $L^{\infty}\left(0, \infty ; L_{p e r}^{2}\left(\Omega ; \mathbb{R}^{3}\right)\right)$,

(iv) there exists a subsequence, still denoted $\left\{\omega_{n}\right\}$, which converges weak-star in $L^{\infty}(0, \infty$; $\left.L_{p e r}^{q}(\Omega ; \mathbb{R})\right)$, for all $1<q \leq p$, to a limit $\omega$.

We claim that $\omega$ is a weak solution of the vorticity equation. To see this, we observe that $\omega_{n}$ satisfies (3.16), for all $n \in \mathbb{N}$, and, up to a subsequence, $\omega_{n} \stackrel{*}{\rightarrow} \omega$ in $L^{\infty}\left(0, \infty ; L_{p e r}^{q}(\Omega ; \mathbb{R})\right)$. Clearly, the linear terms of (3.16) converge, as $n \rightarrow \infty$. Hence, to obtain that $\omega$ satisfies (3.16) it is enough to prove that the nonlinear term of (3.16) converges, as $n \rightarrow \infty$, and that the following identity holds true

$\lim _{n \rightarrow \infty} \int_{0}^{\infty} \int_{\Omega} \int_{\Omega} \mathcal{H}_{\psi}(t, x, y) \omega_{n}(t, y) \omega_{n}(t, x) d y d x d t=\int_{0}^{\infty} \int_{\Omega} \int_{\Omega} \mathcal{H}_{\psi}(t, x, y) \omega(t, y) \omega(t, x) d y d x d t$. 
The remainder of the proof consists in showing that we can pass to the limit in the nonlinear term, in order to establish this identity. Let $\psi \in \mathcal{C}_{c, p e r}^{\infty}([0, \infty) \times \Omega ; \mathbb{R})$ and fix $\rho>0$ such that supp $\psi \subset C_{\rho}$, where $C_{\rho}=\left\{x \in \mathbb{R}^{3}:|\tilde{x}| \leq \rho\right.$ and $\left.\left|x_{3}\right| \leq \pi \kappa\right\}$. Define

$$
\alpha_{n}:=\frac{1}{16 \pi^{3} \kappa^{3}} \int_{C_{\rho}} \omega_{n}(t, x) \partial_{3} \psi(x) d x \text { and } \alpha:=\frac{1}{16 \pi^{3} \kappa^{3}} \int_{C_{\rho}} \omega(t, x) \partial_{3} \psi(x) d x,
$$

and observe that $\lim _{n \rightarrow \infty} \alpha_{n}=\alpha$.

Fix $0<\delta \ll 1$ and $R \gg 2 \max \{\rho, 2 \pi \kappa\}$ and let $\varphi_{\delta}:[0, \infty) \rightarrow[0,1]$ and $\zeta_{R}: \Omega \rightarrow[0,1]$ be smooth cutoff functions, so that

$$
\begin{array}{rr}
\varphi_{\delta}(r) \equiv 1, & r \leq \delta, \\
0 \leq \varphi_{\delta}(r) \leq 1, & \delta<r \leq 2 \delta \\
\varphi_{\delta}(r) \equiv 0, & r>2 \delta ; \\
\zeta_{R}(x) \equiv 1, & |x| \leq R, \\
0 \leq \zeta_{R}(x) \leq 1, & R<|x| \leq 2 R, \\
\zeta_{R}(x) \equiv 0, & |x|>2 R .
\end{array}
$$

Using this notation we have the following decomposition:

$$
\begin{gathered}
\int_{\Omega} \int_{\Omega} \mathcal{H}_{\psi}(t, x, y) \omega_{n}(t, y) \omega_{n}(t, x) d y d x=\int_{\Omega} \int_{\Omega} \mathcal{H}_{\psi}(t, x, y) \varphi_{\delta}(|x-y|) \omega_{n}(t, y) \omega_{n}(t, x) d y d x \\
+\int_{\Omega} \int_{\Omega} \mathcal{H}_{\psi}(t, x, y)\left(1-\varphi_{\delta}(|x-y|)\right) \omega_{n}(t, y) \omega_{n}(t, x) d y d x \equiv I_{1}^{n}+I_{2}^{n} .
\end{gathered}
$$

Using the definition of $\mathcal{H}_{\psi}$ and the Generalized Young Inequality with $1 / s+1 / p=1+1 / p^{\prime}$, we obtain that

$$
\begin{gathered}
\left|I_{1}^{n}\right| \leq \int_{\Omega} \int_{\Omega}\left|\mathcal{H}_{\psi}(t, x, y)\right| \varphi_{\delta}(|x-y|)\left|\omega_{n}(t, y)\right|\left|\omega_{n}(t, x)\right| d y d x \\
\leq C \int_{\Omega} \int_{\Omega}\left(\frac{1}{|x-y|}+\frac{1}{|\tilde{x}-\tilde{y}|}+1\right) \varphi_{\delta}(|x-y|)\left|\omega_{n}(t, y) \| \omega_{n}(t, x)\right| d y d x \\
\leq C\left\|\left(\frac{1}{|\cdot|}+\frac{1}{|\cdot|}+1\right) \varphi_{\delta}(|\cdot|)\right\|_{L^{s}}\left\|\omega_{n}(t, \cdot)\right\|\left\|_{p}\right\| \omega_{n}(t, .) \|_{p} .
\end{gathered}
$$

If $s<2$ then we find that

$$
\left\|\left(\frac{1}{|\cdot|}+\frac{1}{|\tilde{\mid}|}+1\right) \varphi_{\delta}(|\cdot|)\right\|_{L^{s}}^{s} \leq C \delta^{2-s} .
$$

Recall that $\left\|\omega_{n}(t, \cdot)\right\|_{p} \leq C$. Recall, also, that we assumed that $p>4 / 3$, so that $p^{\prime}<4$. Now, since $1 / s+1 / p=1+1 / p^{\prime}$ we have that $s=p^{\prime} / 2$. Therefore we have, indeed, $s<2$ and, hence, $I_{1}^{n} \leq C \delta^{(2-s) / s}=o(1)$ as $\delta \rightarrow 0$.

Next we estimate $I_{2}^{n}$. Since $\int_{\Omega} \omega_{n}(t, x) d x=0$, we can rewrite $I_{2}^{n}$ as

$$
I_{2}^{n}=\int_{\Omega}\left(\int_{\Omega} \mathcal{H}_{\psi}(t, x, y)\left(1-\varphi_{\delta}(|x-y|)\right) \omega_{n}(t, y) d y+\alpha_{n}\right) \omega_{n}(t, x) d x .
$$

Now, we decompose $I_{2}^{n}$ as

$$
\begin{gathered}
I_{2}^{n}=\int_{\Omega}\left(\int_{\Omega} \mathcal{H}_{\psi}(t, x, y)\left(1-\varphi_{\delta}(|x-y|)\right) \omega_{n}(t, y) d y+\alpha_{n}\right) \zeta_{R}(x) \omega_{n}(t, x) d x \\
+\int_{\Omega}\left(\int_{\Omega} \mathcal{H}_{\psi}(t, x, y)\left(1-\varphi_{\delta}(|x-y|)\right) \omega_{n}(t, y) d y+\alpha_{n}\right)\left(1-\zeta_{R}(x)\right) \omega_{n}(t, x) d x \equiv I_{21}^{n}+I_{22}^{n} .
\end{gathered}
$$


We start by estimating $I_{21}^{n}$. We decompose the integral as

$$
\begin{gathered}
I_{21}^{n}=\int_{\Omega}\left(\int_{\Omega} \mathcal{H}_{\psi}(t, x, y)\left(1-\varphi_{\delta}(|x-y|)\right) \zeta_{R}(y) \omega_{n}(t, y) d y\right) \zeta_{R}(x) \omega_{n}(t, x) d x \\
+\int_{\Omega}\left(\int_{\Omega} \mathcal{H}_{\psi}(t, x, y)\left(1-\varphi_{\delta}(|x-y|)\right)\left(1-\zeta_{R}(y)\right) \omega_{n}(t, y) d y+\alpha_{n}\right) \zeta_{R}(x) \omega_{n}(t, x) d x \equiv J_{1}^{n}+J_{2}^{n} .
\end{gathered}
$$

We begin by analyzing $J_{1}^{n}$. Observe that the function $\mathcal{H}_{\psi}(t, x, y)\left(1-\varphi_{\delta}(|x-y|)\right) \zeta_{R}(y) \zeta_{R}(x) \in$ $C_{c}^{\infty}([0, \infty) \times \Omega \times \Omega)$. Therefore we can pass to the limit as $n \rightarrow \infty$ and obtain that

$$
\lim _{n \rightarrow \infty} J_{1}^{n}=\int_{\Omega} \int_{\Omega} \mathcal{H}_{\psi}(t, x, y)\left(1-\varphi_{\delta}(|x-y|)\right) \zeta_{R}(y) \zeta_{R}(x) \omega(t, y) \omega(t, x) d y d x .
$$

Next, we consider $J_{2}^{n}$. Observe that the integrand in the inner integral, with respect to $y$, vanishes for $|y| \leq R$. Also, for $y \in C_{\rho}^{c}=\mathbb{R}^{3} \backslash C_{\rho}$, we have that $\mathcal{H}_{\psi}(t, x, y)=1 /(2 \kappa) \mathcal{K}(x-$ $y) \cdot(\xi(y) \times \nabla \psi(t, x))$. Moreover, we note that $\nabla \psi(x)$ vanishes for all $x \in C_{\rho}^{c}$. Therefore, it is enough to estimate $\mathcal{H}_{\psi}$ for $|y|>R$ and $x \in C_{\rho}$. In this case, $|x-y| \geq|y|-|x| \geq|y| / 2$ and $|\tilde{x}-\tilde{y}| \geq|x-y|-2 \pi \kappa \geq|y| / 4$. Thus, using the notation $\mathcal{K}_{1}$ and $\mathcal{K}_{2}$, introduced in the proof of Lemma 3.2 we have

$$
\mathcal{H}_{\psi}(t, x, y)=\frac{1}{2 \kappa} \mathcal{K}_{1}(x-y) \cdot(\xi(y) \times \nabla \psi(t, x))-\frac{1}{2 \kappa} \mathcal{K}_{2}(x-y) \cdot(\xi(y) \times \nabla \psi(t, x)) .
$$

Introduce $G_{1}:=\frac{1}{2 \kappa} \mathcal{K}_{1}(x-y) \cdot(\xi(y) \times \nabla \psi(t, x))$ and recall the definition of $\mathcal{K}_{2}$ to get that

$$
\begin{gathered}
\mathcal{H}_{\psi}(t, x, y)=G_{1}-\frac{1}{16 \pi^{3} \kappa^{3}} \frac{(\tilde{x}-\tilde{y}, 0)}{|\tilde{x}-\tilde{y}|^{2}} \cdot(\xi(y) \times \nabla \psi(t, x)) \\
=G_{1}-\frac{1}{16 \pi^{3} \kappa^{3}} \frac{1}{|\tilde{x}-\tilde{y}|^{2}}\left((\tilde{x}-\tilde{y}, 0) \cdot(-\tilde{y}, 0) \partial_{3} \psi(t, x)+\kappa(\tilde{x}-\tilde{y}, 0) \cdot\left(-\partial_{2} \psi(t, x), \partial_{1} \psi(t, x), 0\right)\right) \\
=G_{1}-\frac{1}{16 \pi^{3} \kappa^{3}} \frac{1}{|\tilde{x}-\tilde{y}|^{2}}\left(|\tilde{x}-\tilde{y}|^{2} \partial_{3} \psi(t, x)+(\tilde{x}-\tilde{y}, 0) \cdot(-\tilde{x}, 0) \partial_{3} \psi(t, x)\right. \\
\left.+\kappa(\tilde{x}-\tilde{y}, 0) \cdot\left(-\partial_{2} \psi(t, x), \partial_{1} \psi(t, x), 0\right)\right) .
\end{gathered}
$$

Let $G_{2}:=-\frac{1}{16 \pi^{3} \kappa^{3}|\tilde{x}-\tilde{y}|^{2}}\left(-(\tilde{x}-\tilde{y}, 0) \cdot(\tilde{x}, 0) \partial_{3} \psi(t, x)+\kappa(\tilde{x}-\tilde{y}, 0) \cdot\left(-\partial_{2} \psi(t, x), \partial_{1} \psi(t, x), 0\right)\right)$. With this notation we now have

Observe that

$$
\mathcal{H}_{\psi}(t, x, y)=G_{1}-\frac{1}{16 \pi^{3} \kappa^{3}} \partial_{3} \psi(t, x)+G_{2} .
$$

We rewrite $J_{2}^{n}$ as

$$
\left|G_{1}\right| \leq C \frac{1}{|\tilde{x}-\tilde{y}|^{2}}|y| \leq C \frac{1}{|y|} \text { and }\left|G_{2}\right| \leq C \frac{1}{|y|}
$$

$$
\begin{aligned}
J_{2}^{n} & =\int_{\Omega}\left(\int_{\Omega}\left(G_{1}+G_{2}\right) \zeta_{R}(x) \omega_{n}(t, x) d x\right)\left(1-\zeta_{R}(y)\right) \omega_{n}(t, y) d y \\
& -\int_{\Omega} \alpha_{n}\left(1-\zeta_{R}(y)\right) \omega_{n}(t, y) d y+\alpha_{n} \int_{\Omega} \zeta_{R}(x) \omega_{n}(t, x) d x .
\end{aligned}
$$

Using (4.3) we obtain the following estimate

$$
\left|J_{2}^{n}\right| \leq \frac{C}{R}\left\|\omega_{n}(t, .)\right\|_{1}^{2}+2\left|\alpha_{n} \int_{\Omega} \zeta_{R}(x) \omega_{n}(t, x) d x\right| \leq \frac{C}{R}+C\left|\int_{\Omega} \zeta_{R}(x) \omega_{n}(t, x) d x\right| .
$$

It is clear that

$$
\lim _{n \rightarrow \infty} \int_{\Omega} \zeta_{R}(x) \omega_{n}(t, x) d x=\int_{\Omega} \zeta_{R}(x) \omega(t, x) d x .
$$

Moreover, using the Dominated Convergence theorem we obtain that

$$
\lim _{R \rightarrow \infty} \int_{\Omega} \zeta_{R}(x) \omega(t, x) d x=\int_{\Omega} \omega(t, x) d x=0 .
$$

Thus, $\lim _{R \rightarrow \infty} \lim _{n \rightarrow \infty}\left|J_{2}^{n}\right|=0$. 
Next we treat $I_{22}^{n}$. In order to estimate $I_{22}^{n}$, we observe that, since $R \gg 2 \rho$, it follows that $\mathcal{H}_{\psi}(t, x, y)=0$ for all $|x|>R$ and $y \in C_{\rho}^{c}$. Furthermore, for $|x|>R$ and $y \in C_{\rho}$ we have that $|x-y| \geq|x| / 2$ and $|\tilde{x}-\tilde{y}|=|x-y|-2 \pi \kappa \geq|x| / 4$. Hence, $1-\varphi_{\delta}(|x-y|)=1$ for all $|x|>R$ and $y \in C_{\rho}$. In this region we can rewrite $\mathcal{H}_{\psi}$ as

$$
\begin{gathered}
\mathcal{H}_{\psi}(t, x, y)=\frac{1}{2 \kappa}\left(\mathcal{K}_{1}(x-y)-\mathcal{K}_{2}(x-y)\right) \cdot(\xi(y) \times(-\nabla \psi(t, y))-(\xi(x)-\xi(y)) \times \nabla \psi(t, y)) \\
=\frac{1}{2 \kappa}\left(\mathcal{K}_{1}(x-y) \cdot(-\xi(y) \times \nabla \psi(t, y)-(\xi(x)-\xi(y)) \times \nabla \psi(t, y))+\mathcal{K}_{2}(x-y) \cdot(\xi(y) \times \nabla \psi(t, y))\right) \\
+\frac{1}{2 \kappa} \mathcal{K}_{2}(x-y) \cdot((\xi(x)-\xi(y)) \times \nabla \psi(t, y)) \equiv F_{1}+F_{2} .
\end{gathered}
$$

Observe that, for $|x|>R$ and $y \in C_{\rho}$, we get

$$
\left|F_{1}\right| \leq C \frac{1}{|\tilde{x}-\tilde{y}|^{2}}(|y|+|\tilde{x}-\tilde{y}|)+C \frac{1}{|\tilde{x}-\tilde{y}|}|y| \leq C\left(\frac{1}{R^{2}}+\frac{1}{R}\right),
$$

while $F_{2}=-\frac{1}{16 \pi^{3} \kappa^{3}} \partial_{3} \psi(t, y)$, so that $\int_{C_{\rho}} F_{2} \omega_{n}(t, y) d y=-\alpha_{n}$. Thus,

$$
\begin{gathered}
\left|I_{22}^{n}\right| \leq \int_{\Omega} \int_{C_{\rho}}\left|F_{1}\right|\left|\omega_{n}(t, y)\right|\left|\left(1-\zeta_{R}(x)\right)\right|\left|\omega_{n}(t, x)\right| d y d x \\
+\left|\int_{\Omega}\left(\int_{C_{\rho}} F_{2} \omega_{n}(t, y) d y+\alpha_{n}\right)\left(1-\zeta_{R}(x)\right) \omega_{n}(t, x) d x\right| \\
\leq \int_{\Omega} \int_{C_{\rho}} C\left(\frac{1}{R^{2}}+\frac{1}{R}\right)\left|\omega_{n}(t, y) \|\left(1-\zeta_{R}(x)\right)\right|\left|\omega_{n}(t, x)\right| d y d x \\
\leq C\left(\frac{1}{R^{2}}+\frac{1}{R}\right)\left\|\omega_{n}(t, \cdot)\right\|_{1}^{2} \leq C\left(\frac{1}{R^{2}}+\frac{1}{R}\right) \leq \frac{C}{R},
\end{gathered}
$$

if $R>>1$.

We can now pass to the limit as $n \rightarrow \infty$ and use all the estimates obtained to get

$$
\begin{gathered}
\lim _{n \rightarrow \infty} \int_{\Omega} \int_{\Omega} \mathcal{H}_{\psi}(t, x, y) \omega_{n}(t, y) \omega_{n}(t, x) d y d x=\lim _{n \rightarrow \infty}\left(I_{1}^{n}+J_{1}^{n}+J_{2}^{n}+I_{22}^{n}\right) \\
=C \delta^{(2-s) / s}+\lim _{n \rightarrow \infty} \int_{\Omega} \int_{\Omega} \mathcal{H}_{\psi}(t, x, y)\left(1-\varphi_{\delta}(|x-y|)\right) \zeta_{R}(y) \zeta_{R}(x) \omega_{n}(t, y) \omega_{n}(t, x) d y d x \\
\quad+\left(\frac{C}{R}+C \lim _{n \rightarrow \infty}\left|\int_{\Omega} \omega_{n}(t, x) \zeta_{R}(x) d x\right|\right)+\frac{C}{R} \\
=\int_{\Omega} \int_{\Omega} \mathcal{H}_{\psi}(t, x, y)\left(1-\varphi_{\delta}(|x-y|)\right) \zeta_{R}(y) \zeta_{R}(x) \omega(t, y) \omega(t, x) d y d x \\
+C \delta^{(2-s) / s}+\frac{C}{R}+C\left|\int_{\Omega} \omega(t, x) \zeta_{R}(x) d x\right| .
\end{gathered}
$$

Now, let us pass to the limit as $R \rightarrow \infty$ and $\delta \rightarrow 0$ to obtain that

$$
\begin{gathered}
\lim _{n \rightarrow \infty} \int_{\Omega} \int_{\Omega} \mathcal{H}_{\psi}(t, x, y) \omega_{n}(t, y) \omega_{n}(t, x) d y d x \\
=\lim _{R \rightarrow \infty, \delta \rightarrow 0}\left(\int_{\Omega} \int_{\Omega} \mathcal{H}_{\psi}(t, x, y)\left(1-\varphi_{\delta}(|x-y|)\right) \zeta_{R}(y) \zeta_{R}(x) \omega(t, y) \omega(t, x) d y d x\right) \\
+\lim _{R \rightarrow \infty, \delta \rightarrow 0}\left(C \delta^{(2-s) / s}+\frac{C}{R}+C\left|\int_{\Omega} \omega(t, x) \zeta_{R}(x) d x\right|\right) \\
=\lim _{R \rightarrow \infty, \delta \rightarrow 0}\left(\int_{\Omega} \int_{\Omega} \mathcal{H}_{\psi}(t, x, y)\left(1-\varphi_{\delta}(|x-y|)\right) \zeta_{R}(y) \zeta_{R}(x) \omega(t, y) \omega(t, x) d y d x\right) .
\end{gathered}
$$

On the other hand we have that

$$
\int_{\Omega} \int_{\Omega} \mathcal{H}_{\psi}(t, x, y) \omega(t, y) \omega(t, x) d y d x=\int_{\Omega} \int_{\Omega} \mathcal{H}_{\psi}(t, x, y) \varphi_{\delta}(|x-y|) \omega(t, y) \omega(t, x) d y d x
$$




$$
\begin{gathered}
+\int_{\Omega} \int_{\Omega} \mathcal{H}_{\psi}(t, x, y)\left(1-\varphi_{\delta}(|x-y|)\right) \zeta_{R}(y) \zeta_{R}(x) \omega(t, y) \omega(t, x) d y d x \\
+\int_{\Omega}\left(\int_{\Omega} \mathcal{H}_{\psi}(t, x, y)\left(1-\varphi_{\delta}(|x-y|)\right)\left(1-\zeta_{R}(y)\right) \omega(t, y) d y+\alpha\right) \zeta_{R}(x) \omega(t, x) d x \\
+\int_{\Omega}\left(\int_{\Omega} \mathcal{H}_{\psi}(t, x, y)\left(1-\varphi_{\delta}(|x-y|)\right) \omega(t, y) d y+\alpha\right)\left(1-\zeta_{R}(x)\right) \omega(t, x) d x
\end{gathered}
$$

using the same idea from the estimates obtained for the sequence $\omega_{n}$,

Therefore,

$$
\begin{gathered}
=C \delta^{(2-s) / s}+\int_{\Omega} \int_{\Omega} \mathcal{H}_{\psi}(t, x, y)\left(1-\varphi_{\delta}(|x-y|)\right) \zeta_{R}(y) \zeta_{R}(x) \omega(t, y) \omega(t, x) d y d x+ \\
+\left(\frac{C}{R}+C\left|\int_{\Omega} \omega(t, x) \zeta_{R}(x) d x\right|\right)+\frac{C}{R}
\end{gathered}
$$

$$
\begin{gathered}
\int_{\Omega} \int_{\Omega} \mathcal{H}_{\psi}(t, x, y) \omega(t, y) \omega(t, x) d y d x \\
=\lim _{R \rightarrow \infty, \delta \rightarrow 0} \int_{\Omega} \int_{\Omega} \mathcal{H}_{\psi}(t, x, y)\left(1-\varphi_{\delta}(|x-y|)\right) \zeta_{R}(y) \zeta_{R}(x) \omega(t, y) \omega(t, x) d y d x .
\end{gathered}
$$

Finally, we conclude that

$$
\lim _{n \rightarrow \infty} \int_{\Omega} \int_{\Omega} \mathcal{H}_{\psi}(t, x, y) \omega_{n}(t, y) \omega_{n}(t, x) d y d x=\int_{\Omega} \int_{\Omega} \mathcal{H}_{\psi}(t, x, y) \omega(t, y) \omega(t, x) d y d x .
$$

It is clear from the a priori estimates that $\left\{u_{n}\right\}$ is uniformly bounded in $L^{\infty}\left(0, \infty ; L_{p e r}^{2}\left(\Omega ; \mathbb{R}^{3}\right)\right)$ so that there exists a subsequence, which we still denote by $\left\{u_{n}\right\}$, that converges to $u \in L^{\infty}\left(0, \infty ; L_{\text {per }}^{2}\left(\Omega ; \mathbb{R}^{3}\right)\right)$. It remains to prove that the limit $u$ is the velocity associated with the vorticity $\omega$, that is, $u(t, x)=\int_{\Omega} \mathcal{K}(x-y) \times(\xi(y) / \kappa) \omega(t, y) d y$.

Fix $R>0$ and set $U_{R}=\{x \in \Omega:|\tilde{x}| \leq R\}$. Let us fix $\delta>0$ such that $0<\delta \ll R$ and let $\varphi_{\delta}$ be as defined in (4.1), so that

$$
\begin{gathered}
\left\|u_{n}(t, \cdot)-u(t, \cdot)\right\|_{L^{2}\left(U_{R}\right)}^{2}=\int_{U_{R}}\left|\int_{\Omega} \mathcal{K}(x-y) \times \frac{\xi(y)}{\kappa}\left(\omega_{n}(t, y)-\omega(t, y)\right) d y\right|^{2} d x \\
\leq 2 \int_{U_{R}}\left|\int_{\Omega} \varphi_{\delta}(|x-y|) \mathcal{K}(x-y) \times \frac{\xi(y)}{\kappa}\left(\omega_{n}(t, y)-\omega(t, y)\right) d y\right|^{2} d x \\
+2 \int_{U_{R}}\left|\int_{\Omega}\left(1-\varphi_{\delta}(|x-y|)\right) \mathcal{K}(x-y) \times \frac{\xi(y)}{\kappa}\left(\omega_{n}(t, y)-\omega(t, y)\right) d y\right|^{2} d x \equiv \mathcal{I}_{1}^{n}+\mathcal{I}_{2}^{n} .
\end{gathered}
$$

We start by estimating the term $\mathcal{I}_{1}^{n}$. Observe that for $|x| \leq R$ and $|x-y|<2 \delta$ it holds that $|\xi(y)| \leq|x-y|+|x| \leq 2 \delta+R \leq 2 R$ and hence

$$
\begin{gathered}
\mathcal{I}_{1}^{n} \leq C(R) \int_{U_{R}}\left(\int_{\Omega}\left|\varphi_{\delta}(|x-y|)\|\mathcal{K}(x-y)\| \omega_{n}(t, y)-\omega(t, y)\right| d y\right)^{2} d x \\
\leq C(R)\left\|\varphi_{\delta} \mathcal{K}\right\|_{L^{q}}^{2}\left\|\omega_{n}-\omega\right\|_{L^{p}}^{2},
\end{gathered}
$$

where in the last inequality we used the Generalized Young inequality with $1+1 / 2=1 / q+1 / p$. Since $\left\|\varphi_{\delta} \mathcal{K}\right\|_{L^{q}} \leq C \delta^{3-2 q}$ and $\left\|\omega_{n}-\omega\right\|_{L^{p}} \leq\left\|\omega_{n}\right\|_{L^{p}}+\|\omega\|_{L^{p}} \leq C$ then $\mathcal{I}_{1}^{n} \leq C \delta^{3-2 q}$, for all $n \in \mathbb{N}$. It is clear that the condition $p>4 / 3$ implies $q<3 / 2$. Therefore,

$$
\lim _{\delta \rightarrow 0} \lim _{n \rightarrow \infty} \mathcal{I}_{1}^{n}=0 .
$$

Now, we will estimate $\mathcal{I}_{2}^{n}$. Recall the notation introduced in Lemma 3.2, $\mathcal{K}=\mathcal{K}_{1}-\mathcal{K}_{2}$, so

$$
\begin{gathered}
\mathcal{I}_{2}^{n} \leq 4 \int_{U_{R}}\left|\int_{\Omega}\left(1-\varphi_{\delta}(|x-y|)\right) \mathcal{K}_{1}(x-y) \times \frac{\xi(y)}{\kappa}\left(\omega_{n}(t, y)-\omega(t, y)\right) d y\right|^{2} d x \\
+4 \int_{U_{R}}\left|\int_{\Omega}\left(1-\varphi_{\delta}(|x-y|)\right) \mathcal{K}_{2}(x-y) \times \frac{\xi(y)}{\kappa}\left(\omega_{n}(t, y)-\omega(t, y)\right) d y\right|^{2} d x \equiv \mathcal{F}_{n}^{1}+\mathcal{F}_{n}^{2} .
\end{gathered}
$$


We will see that $\mathcal{F}_{n}^{1}$ and $\mathcal{F}_{n}^{2}$ converge to zero when $n \rightarrow \infty$. We start with $\mathcal{F}_{n}^{1}$. Observe that for $|x-y|>\delta$ we have

$$
\begin{aligned}
& \left|\mathcal{K}_{1}(x-y) \times \xi(y)\right|=\left|\mathcal{K}_{1}(x-y) \times(\xi(y)-\xi(x)+\xi(x))\right| \\
\leq & C \frac{1}{|\tilde{x}-\tilde{y}|^{2}}(|\tilde{y}-\tilde{x}|+|\xi(x)|) \leq C(R)\left(\frac{1}{|\tilde{x}-\tilde{y}|}+\frac{1}{|\tilde{x}-\tilde{y}|^{2}}\right) .
\end{aligned}
$$

Thus, for every $x \in U_{R}$ fixed, $\left(1-\varphi_{\delta}(|x-\cdot|)\right) \mathcal{K}_{1}(x-\cdot) \times \xi(\cdot) \in L^{r}(\Omega)$, for all $r>2$. Moreover, since $\left\{\omega_{n}\right\}$ is uniformly bounded in $L^{\infty}\left(0, \infty ; L^{q}(\Omega)\right)$ for all $1 \leq q \leq p$ we obtain that $\omega_{n}(t, \cdot) \stackrel{*}{\rightarrow} \omega(t, \cdot)$ in $L^{q}(\Omega)$, for all $1<q \leq p$, so that we can choose $q$ in such a way that $1<q \leq p$ and $q^{\prime}>2$. Therefore, we obtain that $\lim _{n \rightarrow \infty} \int_{\Omega}\left(1-\varphi_{\delta}(|x-y|)\right) \mathcal{K}_{1}(x-y) \times(\xi(y) / \kappa)\left(\omega_{n}(t, y)-\omega(t, y)\right) d y=0$ for every $x \in U_{R}$. Moreover, we have that

$$
\begin{aligned}
& \left|\int_{\Omega}\left(1-\varphi_{\delta}(|x-y|)\right) \mathcal{K}_{1}(x-y) \times \frac{\xi(y)}{\kappa}\left(\omega_{n}(t, y)-\omega(t, y)\right) d y\right| \\
\leq & C\left\|\left(1-\varphi_{\delta}(|x-\cdot|)\right) \mathcal{K}_{1}(x-\cdot) \times \xi\right\|_{L^{q^{\prime}}}\left\|\omega_{n}(t, \cdot)-\omega(t, \cdot)\right\|_{L^{q}} \leq C_{\delta},
\end{aligned}
$$

where $1 / q+1 / q^{\prime}=1, q \leq p$ and $q^{\prime}>2$, so that the last sequence is uniformly bounded in $n$ by a constant which is an integrable function in $U_{R}$. Thus, we can apply the Dominated Convergence theorem to obtain that $\lim _{n \rightarrow \infty} \mathcal{F}_{n}^{1}=0$.

Now we will prove the convergence of the term $\mathcal{F}_{n}^{2}$. Fix $M>0, M \gg R$ and consider $\zeta_{M}$ as defined in (4.2). Let us split the integral in the following way:

$$
\begin{gathered}
\int_{\Omega}\left(1-\varphi_{\delta}(|x-y|)\right) \zeta_{M}(y) \mathcal{K}_{2}(x-y) \times \frac{\xi(y)}{\kappa}\left(\omega_{n}(t, y)-\omega(t, y)\right) d y \\
+\int_{\Omega}\left(1-\varphi_{\delta}(|x-y|)\right)\left(1-\zeta_{M}(y)\right) \mathcal{K}_{2}(x-y) \times \frac{\xi(y)}{\kappa}\left(\omega_{n}(t, y)-\omega(t, y)\right) d y \equiv \mathcal{J}_{n}^{1}(t, x)+\mathcal{J}_{n}^{2}(t, x) .
\end{gathered}
$$

In order to prove the convergence of $\mathcal{J}_{n}^{1}$, first we recall that $\left\|\omega_{n}(t, \cdot)\right\|_{L^{1}} \leq C$ implies that $\lim _{n \rightarrow \infty} \int_{\Omega} \omega_{n}(t, y) f(y) d y=\int_{\Omega} \omega(t, y) f(y) d y$ for all $f \in C_{0}(\Omega)$. Then, observe that $f_{x}(y):=$ $\left(1-\varphi_{\delta}(|x-y|)\right) \zeta_{M}(y) \mathcal{K}_{2}(x-y) \times(\xi(y) / \kappa) \in \mathcal{C}_{0}(\Omega)$ for all $x \in U_{R}$, thus

$$
\mathcal{J}_{n}^{1}(t, x)=\int_{\Omega} f_{x}(y)\left(\omega_{n}(t, y)-\omega(t, y)\right) d y \rightarrow 0 \text { as } n \rightarrow \infty .
$$

Finally, observe that

$$
\begin{gathered}
\mathcal{K}_{2}(x-y) \times \frac{\xi(y)}{\kappa}=\frac{1}{16 \pi^{3} \kappa^{3}} \frac{1}{|\tilde{x}-\tilde{y}|^{2}}(\tilde{x}-\tilde{y}, 0) \times(\xi(y)-\xi(x)+\xi(x)) \\
=\frac{1}{16 \pi^{3} \kappa^{3}}(0,0,1)+\frac{1}{16 \pi^{3} \kappa^{3}} \frac{1}{|\tilde{x}-\tilde{y}|^{2}}(\tilde{x}-\tilde{y}, 0) \times \xi(x) .
\end{gathered}
$$

Moreover, since $x \in U_{R}$ then $\left(1-\varphi_{\delta}(x-y)\right)\left(1-\zeta_{M}(y)\right)=\left(1-\zeta_{M}(y)\right)$. Thus,

$$
\begin{aligned}
& \left|\mathcal{J}_{n}^{2}(t, x)\right| \leq\left|\frac{1}{16 \pi^{3} \kappa^{3}} \int_{\Omega}\left(1-\zeta_{M}(y)\right)\left(\omega_{n}(t, y)-\omega(t, y)\right) d y\right| \\
& \quad+C \int_{\Omega}\left(1-\zeta_{M}(y)\right) \frac{1}{|\tilde{x}-\tilde{y}|}\left(\left|\omega_{n}(t, y)\right|+|\omega(t, y)|\right) d y \\
& \leq\left|\frac{1}{16 \pi^{3} \kappa^{3}} \int_{\Omega}\left(1-\zeta_{M}(y)\right)\left(\omega_{n}(t, y)-\omega(t, y)\right) d y\right|+\frac{C}{M} .
\end{aligned}
$$

Observe that, as $\int_{\Omega} \omega_{n}(t, y) d y=0=\int_{\Omega} \omega(t, y) d y$ and $\zeta_{M} \in L^{p^{\prime}}$, we have

$$
\lim _{n \rightarrow \infty} \int_{\Omega}\left(1-\zeta_{M}(y)\right)\left(\omega_{n}(t, y)-\omega(t, y)\right) d y=-\lim _{n \rightarrow \infty} \int_{\Omega} \zeta_{M}(y)\left(\omega_{n}(t, y)-\omega(t, y)\right) d y=0 .
$$

Thus, $\lim _{n \rightarrow \infty} \mathcal{J}_{n}^{2}(t, x)=\mathcal{O}\left(M^{-1}\right)$. Therefore, we find that

$\lim _{n \rightarrow \infty} \int_{\Omega}\left(1-\varphi_{\delta}(x-y)\right) \mathcal{K}_{2}(x-y) \times \frac{\xi(y)}{\kappa}\left(\omega_{n}(t, y)-\omega(t, y)\right) d y=\lim _{M \rightarrow \infty} \lim _{n \rightarrow \infty}\left(\mathcal{J}_{n}^{1}(t, x)+\mathcal{J}_{n}^{2}(t, x)\right)=0$. 
Furthermore, it follows from the calculations above that

$$
\begin{gathered}
\left|\int_{\Omega}\left(1-\varphi_{\delta}(|x-y|)\right) \mathcal{K}_{2}(x-y) \times \frac{\xi(y)}{\kappa}\left(\omega_{n}(t, y)-\omega(t, y)\right) d y\right| \leq\left|\mathcal{J}_{n}^{1}(t, x)\right|+\left|\mathcal{J}_{n}^{2}(t, x)\right| \\
\leq C\left(\left\|f_{x}\right\|_{L^{\infty}}+\frac{1}{M}+1\right)\left\|\omega_{n}-\omega\right\|_{L^{1}} \leq C_{\delta, M} .
\end{gathered}
$$

Thus, by the Dominated Convergence theorem, we conclude that $\lim _{n \rightarrow \infty} \mathcal{F}_{n}^{2}=0$.

Finally, we end up with

$$
\lim _{n \rightarrow \infty}\left\|u_{n}(t, \cdot)-u(t, \cdot)\right\|_{L^{2}\left(U_{R}\right)}^{2}=\lim _{\delta \rightarrow 0} \lim _{n \rightarrow \infty}\left(\mathcal{I}_{1}^{n}+\mathcal{I}_{2}^{n}\right)=0 .
$$

It is not hard to see that all the estimates used were uniform in $t$, so that

$$
\lim _{n \rightarrow \infty}\left\|u_{n}-u\right\|_{L^{\infty}\left(0, \infty ; L^{2}\left(U_{R}\right)\right)}=0 .
$$

Therefore, $u_{n} \rightarrow u$ in $L^{\infty}\left(0, \infty ; L_{\text {loc,per }}^{2}\left(\Omega, \mathbb{R}^{3}\right)\right)$ hence follows the desired result.

Remark 4.1. We observe that, from the proof of the last theorem, it follows that $u$ is a weak solution of the Euler equations in the velocity formulation. Indeed, since each $u_{n}$ is a smooth solution of the Euler equations, we find that, for all test function $\Phi \in \mathcal{C}_{c, p e r}^{\infty}\left([0, \infty) \times \Omega, \mathbb{R}^{3}\right)$ such that div $\Phi=0$, the following identity holds true:

$\int_{0}^{\infty} \int_{\Omega} \Phi_{t}(t, x) \cdot u_{n}(t, x) d x d t+\int_{0}^{\infty} \int_{\Omega}\left[\left(u_{n}(t, x) \cdot \nabla\right) \Phi(t, x)\right] \cdot u_{n}(t, x) d x d t+\int_{\Omega} \Phi(0, x) \cdot u_{n}(0, x) d x=0$. Now, since $u_{n} \rightarrow u$ in $L^{\infty}\left(0, \infty ; L_{\text {loc,per }}^{2}\left(\Omega, \mathbb{R}^{3}\right)\right.$, we can pass to the limit, as $n \rightarrow \infty$, on the left-hand-side above and obtain that $u$ is a weak solution of the Euler equations.

\section{SECOND EXISTENCE THEOREM: GENERAL CASE}

In this section we will prove that the existence result can also be obtained in the case where the initial vorticity does not have vanishing integral. In order to do so, we must introduce another definition of weak solution of the vorticity equation, adapted to this case.

We can not use the Biot-Savart law in the case where the integral of the vorticity is not zero. In order to deal with this issue we define a new operator which plays the role of the Biot-Savart law. The idea is to decompose the vorticity in two terms, one with zero integral and the other being the vorticity associated with a helical velocity field which is a smooth, radially symmetric, steady solution of the Euler equations. This construction is inspired by the radial-energy decomposition of a velocity field (see [18). The velocity field can then be recovered from the vorticity in the following simple way. We apply the Biot-Savart law to the term which has zero integral and, for the other term, we already have the associated velocity field. With this idea in mind, let us give the following definition:

Definition 5.1. Let $p>4 / 3$. We define the operator

$$
\begin{aligned}
\Xi: L_{p e r}^{p}(\Omega ; \mathbb{R}) \cap L_{\text {per }}^{1}(\Omega ; \mathbb{R}) & \longrightarrow L_{\text {loc,per }}^{2}(\Omega ; \mathbb{R}) \\
\omega & \longmapsto \Xi[\omega]
\end{aligned}
$$

as

$$
\Xi[\omega](x):=\int_{\Omega} \mathcal{K}(x-y) \times \frac{\xi(y)}{\kappa}(\omega(y)-\varphi(|\tilde{y}|)) d y+\bar{u}(x),
$$

where $\varphi:[0, \infty) \rightarrow \mathbb{R}$ is any smooth function with compact support in $(0, \infty)$ such that

$$
\int_{0}^{\infty} \varphi(r) r d r=\int_{\mathbb{R}^{2}} \omega(\tilde{x}, 0) d \tilde{x}
$$

and $\bar{u}$ is defined for all $x \in \Omega$ by

$$
\bar{u}(x)=\left(\frac{\tilde{x}^{\perp}}{|\tilde{x}|^{2}}, \frac{1}{\kappa}\right) \int_{0}^{|\tilde{x}|} \varphi(r) r d r,
$$

where $\tilde{x}^{\perp}=\left(-x_{2}, x_{1}\right)$. 
Remark 5.1. The operator $\Xi$ is well-defined. Indeed, since $\omega \in L_{\text {per }}^{p}(\Omega ; \mathbb{R}) \cap L_{\text {per }}^{1}(\Omega ; \mathbb{R})$ and $\varphi \in C_{c}^{\infty}((0, \infty))$ it is not hard to see that $\mathcal{K} * \xi(\cdot) / \kappa(\omega(\cdot)-\varphi(|\tilde{r}|)) \in L_{\text {loc,per }}^{2}(\Omega ; \mathbb{R})$. Now, let us prove that the definition of $\Xi$ does not depend on the particular choice of $\varphi$. To do so, consider $\varphi, \zeta \in \mathcal{C}^{\infty}([0, \infty))$ with compact support in $(0, \infty)$ and such that

$$
\int_{0}^{\infty} \varphi(r) r d r=\int_{0}^{\infty} \zeta(r) r d r=\int_{\mathbb{R}^{2}} \omega(\tilde{x}, 0) d \tilde{x} .
$$

Set

$$
\bar{u}(x)=\left(\frac{\tilde{x}^{\perp}}{|\tilde{x}|^{2}}, \frac{1}{\kappa}\right) \int_{0}^{|\tilde{x}|} \varphi(r) r d r \text { and } \bar{v}(x)=\left(\frac{\tilde{x}^{\perp}}{|\tilde{x}|^{2}}, \frac{1}{\kappa}\right) \int_{0}^{|\tilde{x}|} \zeta(r) r d r .
$$

Observe that it is enough to prove that

$$
\int_{\Omega} \mathcal{K}(x-y) \times \frac{\xi(y)}{\kappa}(\zeta(|\tilde{y}|)-\varphi(|\tilde{y}|)) d y+\bar{u}(x)-\bar{v}(x)=0 .
$$

First, observe that curl $\bar{u}(y)=\xi(y) / \kappa \varphi(|\tilde{y}|)$ and curl $\bar{v}(y)=\xi(y) / \kappa \zeta(|\tilde{y}|)$. Hence (5.2) is equivalent to

$$
\int_{\Omega} \mathcal{K}(x-y) \times \operatorname{curl}(\bar{v}(y)-\bar{u}(y)) d y=\bar{v}(x)-\bar{u}(x) .
$$

Finally, observe that the vector field defined by $\Phi:=\operatorname{curl}(\bar{v}-\bar{u})$ is such that $\int_{\Omega} \Phi(x) d x=0$, $\operatorname{div} \Phi=0, \Phi \in C_{c, p e r}^{\infty}\left(\Omega ; \mathbb{R}^{3}\right)$ and $|\Phi(x)|$ goes to zero as $|\tilde{x}| \rightarrow \infty$; we can, therefore, use Proposition 3.2 to obtain the identity (5.3).

The definition of weak solution in the case where the vorticity is not balanced follows naturally, and it is stated below:

Definition 5.2. Given $\omega^{0} \in L_{c, p e r}^{p}(\Omega ; \mathbb{R})$, for some $p>4 / 3$, we say that $\omega=\omega(t, x)$ is a weak solution of $\omega_{t}+(u \cdot \nabla) \omega=0$ if

(i) $\omega \in L^{\infty}\left(0, T ; L_{p e r}^{p}(\Omega ; \mathbb{R}) \cap L_{p e r}^{1}(\Omega ; \mathbb{R})\right)$;

(ii) the velocity field $u(t, \cdot)=\Xi[\omega(t, \cdot)]$, for a.e. $t \in[0, T]$;

(iii) for all test function $\psi \in \mathcal{C}_{c}^{\infty}([0, T) \times \Omega ; \mathbb{R})$

$$
\int_{0}^{T} \int_{\Omega} \psi_{t}(t, x) \omega(t, x) d x d t+\int_{0}^{T} \int_{\Omega} u(x, t) \cdot \nabla \psi(x) \omega(t, x) d x d t+\int_{\Omega} \psi(x, 0) \omega^{0}(x) d x=0 .
$$

Next, we state and prove existence of a weak solution for the vorticity equation without the integral constraint on the initial vorticity:

Theorem 5.1. Given $\omega^{0} \in L_{c, p e r}^{p}(\Omega ; \mathbb{R})$, for some $p>4 / 3$, there exists a weak solution $\omega=\omega(t, x)$ of (3.15) in the sense of Definition 5.2.

Proof. We have already proved the theorem in the case $\int \omega^{0} d x=0$. Now, suppose that $\int \omega^{0} d x \neq 0$. Fix $\varphi \in \mathcal{C}^{\infty}([0, \infty))$, with compact support in $(0, \infty)$, such that

$$
\int_{0}^{\infty} \varphi(r) r d r=\int_{\mathbb{R}^{2}} \omega^{0}(\tilde{x}, 0) d \tilde{x}
$$

and define $\tilde{\omega}^{0}=\omega^{0}-\phi$, where $\phi=\phi(x)=\varphi(|\tilde{x}|)$. We introduce an approximating sequence $\tilde{\omega}_{n}^{0}$ given by

$$
\tilde{\omega}_{n}^{0}=\omega_{n}^{0}-\phi,
$$

where $\omega_{n}^{0}$ is the sequence of mollifiers defined by $\omega_{n}^{0}=\rho_{n} * \omega^{0}$. Observe that $\int_{\Omega} \tilde{\omega}_{n}^{0} d x=0$ for all $n$. Thus, for all $n$ we can define the velocity field associated with $\omega_{n}^{0}$ through the Biot-Savart law,

$$
\tilde{u}_{n}^{0}=\mathcal{K} *\left(\tilde{\omega}_{n}^{0} \frac{\xi}{\kappa}\right) .
$$


By Corollary 3.1 we have that, in particular, $\tilde{u}_{n}^{0} \in \mathcal{C}^{\infty}\left(\Omega ; \mathbb{R}^{3}\right) \cap L_{\text {per }}^{2}\left(\Omega ; \mathbb{R}^{3}\right)$ for all $n$. Using the standard energy estimates it is not hard to prove that, for each $n \in \mathbb{N}$, the system

$$
\left\{\begin{array}{l}
\partial_{t} \tilde{u}+(\tilde{u} \cdot \nabla) \tilde{u}+(\tilde{u} \cdot \nabla) \bar{u}+(\bar{u} \cdot \nabla) \tilde{u}+\nabla \tilde{P}=0 \\
\operatorname{div} \tilde{u}=0 \\
\tilde{u}(0, x)=\tilde{u}_{n}^{0}(x) \\
|\tilde{u}|(x) \rightarrow 0 \text { as }|\tilde{x}| \rightarrow \infty .
\end{array}\right.
$$

has a unique solution in $\mathcal{C}^{\infty}\left([0, \infty) \times \Omega ; \mathbb{R}^{3}\right) \cap L^{\infty}\left(0, \infty ; L_{p e r}^{2}\left(\Omega ; \mathbb{R}^{3}\right)\right)$. Let $\tilde{u}_{n} \in \mathcal{C}^{\infty}\left([0, \infty) \times \Omega ; \mathbb{R}^{3}\right) \cap$ $L^{\infty}\left(0, \infty ; L_{\text {per }}^{2}\left(\Omega ; \mathbb{R}^{3}\right)\right)$ be such solution. It is easy to see that $\tilde{u}_{n}^{0}$ is helical and has vanishing helical swirl and, therefore, $\tilde{u}_{n}$ is also helical and has vanishing helical swirl, for all $n \in \mathbb{N}$. Now, set

$$
u_{n}=\tilde{u}_{n}+\bar{u} \quad \text { where } \quad \bar{u}(x)=\left(\frac{\tilde{x}^{\perp}}{|\tilde{x}|^{2}}, \frac{1}{\kappa}\right) \int_{0}^{|\tilde{x}|} \varphi(r) r d r .
$$

We have that the velocity field $u_{n}$ satisfies the following properties:

(i) for each $n, u_{n}$ is a solution of the following problem

$$
\left\{\begin{array}{l}
\partial_{t} u+(u \cdot \nabla) u+\nabla P=0 \\
\operatorname{div} u=0 \\
u(0, x)=\tilde{u}_{n}^{0}(x)+\bar{u}(x) \\
\left|\left(u_{1}, u_{2}\right)\right|(x) \rightarrow 0 \text { and }\left|u_{3}\right|(x) \rightarrow \beta \text { as }|\tilde{x}| \rightarrow \infty
\end{array}\right.
$$

where $\beta=(1 / \kappa)\left|\int_{0}^{\infty} \varphi(r) r d r\right|, P=\tilde{P}+\bar{P}$ and $\bar{P}$ is the pressure associated with the steady solution $\bar{u}$ of the Euler equations;

(ii) $u_{n}$ is helical and has vanishing helical swirl for all $n$;

(iii) denote the third component of curl $u_{n}$ by $\omega_{n}$, then curl $u_{n}=\omega_{n} \xi / \kappa$ for all $n$;

(iv) $u_{n}(t, x)=\Xi \omega_{n}(t, x)=\left(\mathcal{K} *\left(\left(\omega_{n}-\phi\right) \xi / \kappa\right)\right)(t, x)+\bar{u}(x)$.

It is also clear that, for each $n \in \mathbb{N}, \omega_{n} \in C^{\infty}([0, \infty) \times \Omega)$ and $\partial_{t} \omega_{n}+\left(u_{n} \cdot \nabla\right) \omega_{n}=0$. Therefore, for all $1 \leq q \leq p$ we have $\left\|\omega_{n}(t)\right\|_{L^{q}(\Omega)}=\left\|\omega_{n}(0)\right\|_{L^{q}(\Omega)} \leq\left\|\omega^{0}\right\|_{L^{q}} \leq C$, for all $n \in \mathbb{N}$ and $t \geq 0$. Then, there exist a subsequence, which we still denote by $\left\{\omega_{n}\right\}_{n}$, and a limit $\omega \in$ $L^{\infty}\left(0, \infty ; L_{\text {per }}^{p}(\Omega ; \mathbb{R}) \cap L_{\text {per }}^{1}(\Omega ; \mathbb{R})\right)$ such that $\omega_{n} \stackrel{*}{\rightarrow} \omega$ in $L^{\infty}\left(0, \infty ; L^{p}(\Omega ; \mathbb{R})\right)$. Furthermore,

$$
\int_{0}^{T} \int_{\Omega} \psi_{t}(t, x) \omega_{n}(t, x) d x d t+\int_{0}^{T} \int_{\Omega} u_{n}(t, x) \cdot \nabla \psi(x) \omega_{n}(t, x) d x d t+\int_{\Omega} \psi(x, 0) \omega_{n}^{0}(x) d x=0 .
$$

To show that $\omega$ is a weak solution of vorticity equation in the sense of Definition 5.2, we have to prove that we can pass to the limit on the left-hand-side of the identity above, as $n \rightarrow \infty$ : true

$$
\begin{gathered}
\lim _{n \rightarrow \infty}\left\{\int_{0}^{T} \int_{\Omega} \psi_{t}(t, x) \omega_{n}(t, x) d x d t+\int_{0}^{T} \int_{\Omega} u_{n}(t, x) \cdot \nabla \psi(x) \omega_{n}(t, x) d x d t+\int_{\Omega} \psi(x, 0) \omega_{n}^{0}(x) d x\right\}= \\
=\int_{0}^{T} \int_{\Omega} \psi_{t}(t, x) \omega(t, x) d x d t+\int_{0}^{T} \int_{\Omega} u(t, x) \cdot \nabla \psi(x) \omega(t, x) d x d t+\int_{\Omega} \psi(x, 0) \omega^{0}(x) d x .
\end{gathered}
$$

The proof of relation (5.6) follows along the same lines as the proof of Theorem 4.1, using the fact that $u_{n}=\Xi \omega_{n}$.

We conclude with a few final observations. First, the critical regularity for existence of a weak solution in the 2D and axisymmetric case is $p=1$, see 6, 21, 3. We expect helical flows to behave similarly, so that $p>4 / 3$ should not be sharp, just a consequence of the limitations of the estimates available. However, the symmetrization idea that brings the existence result from $p>4 / 3$ to vortex sheets in the 2D case, see [20, has already been used in the proof of Theorem 4.1, so improvements have to come from somewhere else. Some natural open problems are, therefore, proving existence closer to vortex sheet data, seeking an adaptation of Delort's axisymmetric result to helical flows and formulating and studying convergence of helical vortex methods. Another natural line of research is to study the vanishing viscosity limit, which is currently under investigation.

Acknowledgments: Research of H. J. Nussenzveig Lopes is supported in part by CNPq grant \# 306331 / 2010-1, FAPERJ grant \# E-26/103.197/2012. Research of Milton C. Lopes Filho is supported in part by CNPq 
grant \# 303089 / 2010-5. Anne Bronzi's research is supported by Post-Doctoral grant \# 236994/2012-3. This work was supported by FAPESP Thematic Project \# 2007/51490-7 and by the FAPESP grant \# 05/58136-9. The research presented here was part of Anne Bronzi's doctoral dissertation at the mathematics graduate program of UNICAMP.

\section{REFERENCES}

[1] D. Chae. Remarks on the blow-up criterion of the three-dimensional Euler equations. Nonlinearity, 18(3):10211029, 2005.

[2] D. Chae. On the blow-up problem for the axisymmetric 3D Euler equations. Nonlinearity, 21(9):2053-2060, 2008.

[3] D. Chae and O. Y. Imanuvilov. Existence of axisymmetric weak solutions of the 3-D Euler equations for near-vortex-sheet initial data. Electron. J. Differential Equations, (26), 1998.

[4] D. Chae and N. Kim. Axisymmetric weak solutions of the 3-D Euler equations for incompressible fluid flows. Nonlinear Anal., 29(12):1393-1404, 1997.

[5] C. DeLellis and L. Székelyhidi Jr. On admissibility criteria for weak solutions of the Euler equations. Arch. Ration. Mech. Anal., 195(1):225-260, 2010.

[6] J.-M. Delort. Existence de nappes de tourbillon en dimension deux. J. Amer. Math. Soc., 4(3):553-586, 1991.

[7] J.-M. Delort. Une remarque sur le problème des nappes de tourbillon axisymétriques sur $\mathbb{R}^{3}$. J. Funct. Anal., 108(2):274-295, 1992.

[8] A. Dutrifoy. Existence globale en temps de solutions hélicoïdales des équations d'Euler. C. R. Acad. Sci. Paris Sér. I Math., 329(7):653-656, 1999.

[9] B. Ettinger and E. Titi. Global existence and uniqueness of weak solutions of three-dimensional Euler equations with helical symmetry in the absence of vorticity stretching. SIAM J. Math. Anal., 41(1):269-96, 2009.

[10] L. C. Evans. Partial differential equations, volume 19 of Graduate Studies in Mathematics. American Mathematical Society, Providence, RI, 1998.

[11] G. B. Folland. Fourier analysis and its applications. The Wadsworth \& Brooks/Cole Mathematics Series. Wadsworth \& Brooks/Cole Advanced Books \& Software, Pacific Grove, CA, 1992.

[12] S. Gang and X. Zhu. Axisymmetric solutions to the 3D Euler equations. Nonlinear Anal., 66(9):1938-1948, 2007.

[13] Q. Jiu and Z. Xin. On strong convergence to 3D axisymmetric vortex sheets. J. Differential Equations, 233(1):33-50, 2006.

[14] T. Kato. Nonstationay flows of viscous and ideal fluids in $\mathbb{R}^{3}$. J. Funct. Anal., 9:296-305, 1972.

[15] W. Magnus, F. Oberhettinger, and R. P. Soni. Formulas and Theorems for the Special Functions of Mathematical Physics. Springer-Verlag New York, Inc., New York, third edition, 1966.

[16] A. Mahalov, E. S. Titi, and S. Leibovich. Invariant helical subspaces for the Navier-Stokes equations. Arch. Rational Mech. Anal., 112(3):193-222, 1990.

[17] A. Majda. Vorticity and the mathematical theory of incompressible fluid flow. Comm. Pure Appl. Math., 39(S):S187-S220, 1986.

[18] A. Majda and A. Bertozzi. Vorticity and incompressible flow, volume 27 of Cambridge Texts in Applied Mathematics. Cambridge University Press, Cambridge, 2002.

[19] X. Saint Raymond. Remarks on axisymmetric solutions of the incompressible Euler system. Comm. Partial Differential Equations, 19(1-2):321-334, 1994.

[20] S. Schochet. The weak vorticity formulation of the 2-D Euler equations and concentration-cancellation. Comm. Partial Differential Equations, 20(5-6):1077-1104, 1995.

[21] I. Vecchi and S. Wu. On $L^{1}$-vorticity for 2-D incompressible flow. Manuscripta Math., 78(4):403-412, 1993.

[22] E. Wiedemann. Existence of weak solutions for the incompressible Euler equations. Ann. Inst. H. Poincaré Anal. Non Linéaire, 28(5):727-730, 2011.

(Anne C. Bronzi) Deptartment of Mathematics, Statistics and Computer Science, University of IlliNOIS, ChicAGO, IL 60607, U.S.A.

E-mail address: annebronzi@gmail.com

(M. C. Lopes Filho) Instituto de Matemática, Universidade Federal do Rio de Janeiro, Cidade UniverSitária - Ilha do Fundão, Caixa Postal 68530, 21941-909 Rio de Janeiro, RJ - Brasil.

E-mail address: mlopes@im.ufrj.br

(H. J. Nussenzveig Lopes) Instituto de Matemática, Universidade Federal do Rio de Janeiro, Cidade Universitária - Ilha do Fundão, Caixa Postal 68530, 21941-909 Rio de Janeiro, RJ - Brasil.

E-mail address: hlopes@im.ufrj.br 